\title{
Solar coronal-hole plasma densities and temperatures
}

\author{
K. Wilhelm \\ Max-Planck-Institut für Sonnensystemforschung (MPS), 37191 Katlenburg-Lindau, Germany \\ e-mail: wilhelm@mps.mpg.de
}

Received 15 December 2005 / Accepted 13 April 2006

\begin{abstract}
Polar plumes extending from the Sun into the solar corona have long been seen during eclipses, and can now be studied without this restriction with telescopes and spectrometers on board of spacecraft. Despite the large amount of observational data available on this prominent phenomenon, it is not clear whether plumes contribute substantially to the fast solar-wind streams emanating from coronal holes. An understanding of the processes leading to the formation of bright plumes and the surrounding darker inter-plume regions in coronal holes requires a good knowledge of the physical conditions in plumes and their environment. This investigation aims at measuring the electron densities and temperatures in these regions with the help of radiance ratios of ultraviolet emission lines obtained by SUMER on SOHO. It finds densities of about $7 \times 10^{7} \mathrm{~cm}^{-3}$ in bright plumes and $1.3 \times 10^{7} \mathrm{~cm}^{-3}$ in inter-plume lanes at $\approx 45 \mathrm{Mm}$ above the limb. At this height, the total plume cross-section relative to the size of the coronal hole was found to be less than $8 \%$. The densities drop by a factor of roughly two over the next $80 \mathrm{Mm}$ in height, in lanes a little less than seen in plumes. In this height range, the electron temperatures in plumes are $\approx 7.5 \times 10^{5} \mathrm{~K}$ and $\approx 1.13 \times 10^{6} \mathrm{~K}$ in inter-plume regions. The effective ion temperatures, deduced from the line widths, are higher and nearly independent of the altitude in plumes, whereas they increase in inter-plume regions, starting from an even higher level. No systematic dependence of the line-of-sight bulk velocities on the brightness could be found in the coronal-hole plasma.
\end{abstract}

Key words. Sun: UV radiation - Sun: corona - Sun: solar wind

\section{Introduction}

Polar plumes are prominent features of the solar corona. They are observed in coronal holes both in visible and ultraviolet radiation. There is fairly conclusive evidence that the plumes studied in various bands of the electromagnetic spectrum are different manifestations of the same phenomenon (DeForest et al. $1997,2001 \mathrm{a}, \mathrm{b})$. However, there is no undisputed theoretical concept available at this stage, and many observational facts even seem to be in conflict with each other. In particular, the threedimensional structure of plumes and their dynamical properties, along with those of the inter-plume regions are under discussion.

Many studies found indications that plumes are tracers of (open) magnetic field lines (e.g., Lamy et al. 1997; Banaszkiewicz et al. 1998; Lites et al. 1999; Young et al. 1999; DeForest et al. 2001a). There is general agreement on an apparent plume width of $20 \mathrm{Mm}$ to $40 \mathrm{Mm}$ in the low corona (cf., e.g., Banerjee et al. 2000a; Patsourakos \& Vial 2000; Llebaria et al. 2002). Wang et al. (1997) have demonstrated the association of plumes with the magnetic network, the cell structure of which has similar dimensions. The cross-sections of plumes are still a matter of debate, as most observations have been performed above the solar limb, where line-of-sight (LOS) effects make unambiguous measurements perpendicular to the plane of the sky difficult. The relationship between plumes and coronal bright points may provide some insight into this dimension. Wang (1998) and Del Zanna et al. (2003) observed plume/bright point associations that leave little doubt about the limited extent of the depth of plumes along the LOS. However, Gabriel et al. (2003) analysed a bright plume with an improved Dopplerdimming method. They provide evidence that it occupied a considerable fraction of the LOS, and suggest that there might be two types of plumes.

Early plume observations during eclipses and recent ones by spacecraft have led to the conclusion that plumes have lower outflow speeds than inter-plume regions (van de Hulst 1950a,b; Antonucci et al. 1997; Noci et al. 1997; Wilhelm 1999a; Wilhelm et al. 2000; Patsourakos \& Vial 2000; Teriaca et al. 2003), and thus do not appear to be the main contributors to the fast solar-wind streams emanating from coronal holes (Noci 1973; Krieger et al. 1973), whereas other theoretical and observational investigations find higher outflow speeds in plumes than in inter-plume regions, at least at some altitudes above the photosphere (Casalbuoni et al. 1999; Gabriel et al. 2002, 2003, 2005).

Progress in our understanding of the processes that lead to the formation of plumes can only be made if the physical conditions in plumes and inter-plume regions are known in detail. This investigation studies the number densities and temperatures of electrons in a coronal hole at heights from $31 \mathrm{Mm}$ to $159 \mathrm{Mm}$ with the help of the radiance ratios of vacuum-ultraviolet emission lines observed with the Solar Ultraviolet Measurements of Emitted Radiation (SUMER) spectrometer (Wilhelm et al. 1995, 1997; Lemaire et al. 1997) on the Solar and Heliospheric Observatory (SOHO). The widths of the Si VIII $144.6 \mathrm{~nm}$ and $\mathrm{Mg}$ IX $70.6 \mathrm{~nm}$ lines will be characterized as well, from which the effective ion temperatures can be deduced. An attempt is also made to measure the LOS bulk velocities from the observed Doppler shifts of the emission lines. Since the threedimensional configuration of plumes is one of the main questions, it was felt that observations in two spatial dimensions, combined with spectral information, lend themselves more to a successful analysis than do one-dimensional ones. Based on a 
unique data set, only the restricted height range will be treated here, but observations at lower and higher altitudes are also, of course, important (e.g., Fludra et al. 1999; Giordano et al. 2000).

It is assumed that there are two different plasma regimes present along the LOS in coronal holes, namely plumes and inter-plume regions. This assumption is supported by many previous studies (e.g., Antonucci et al. 1997; Noci et al. 1997; Hassler et al. 1997; Wilhelm et al. 1998; Wilhelm 1999b; Lites et al. 1999; Del Zanna et al. 2003). Although there is consensus on whether the electron number density is higher in plumes than in inter-plume regions, the deduced ratios in the low corona vary widely from near unity to about five.

Polar coronal holes are best developed during the minimum of a solar activity cycle. Consequently, many plume and coronalhole studies were carried out under very quiet conditions of the Sun in 1996 and 1997, shortly after the launch of SOHO on 5 December 1995 (DeForest et al. 1997; Hassler et al. 1997; David et al. 1998; Wilhelm et al. 1998; Giordano et al. 2000). Surprisingly low electron temperatures of less than $1 \mathrm{MK}$ were observed and found to be accompanied by much higher ion temperatures, which were somewhat lower in plumes than in interplume regions. The large first-ionization potential (FIP) effect in plumes inferred from Skylab data by Widing \& Feldman (1992) could not be corroborated, but a moderate FIP effect seems to be a persistent feature of plumes (Wilhelm \& Bodmer 1998; Del Zanna \& Bromage 1999; Del Zanna et al. 2003).

Many measurements of the coronal electron density, $n_{\mathrm{e}}$, were performed using the density-sensitive ratio of the forbidden silicon lines $\mathrm{Si}$ VIII $2 \mathrm{~s}^{2} 2 \mathrm{p}^{3}{ }^{4} \mathrm{~S}_{3 / 2}{ }^{2}{ }^{2} \mathrm{D}_{5 / 2}$ and ${ }^{4} \mathrm{~S}_{3 / 2}-{ }^{2} \mathrm{D}_{3 / 2}$ at $144.0 \mathrm{~nm}$ and 144.6 nm (Feldman et al. 1978; Laming et al. 1997; Doschek et al. 1997; Banerjee et al. 1998; Wilhelm et al. 1998; Doyle et al. 1999). This ratio is insensitive to temperature variations. Electron temperatures, $T_{\mathrm{e}}$, in the corona can be measured, among other methods, with the help of the magnesium line ratio of the temperature-sensitive pair $\mathrm{Mg} I X \mathrm{~s}^{2}{ }^{1} \mathrm{~S}_{0}-2 \mathrm{~s} 2 \mathrm{p}{ }^{3} \mathrm{P}_{1}$ and $2 \mathrm{~s} 2 \mathrm{p}{ }^{1} \mathrm{P}_{1}-2 \mathrm{p}^{2}{ }^{1} \mathrm{D}_{2}$ at $70.6 \mathrm{~nm}$ and $75.0 \mathrm{~nm}$, respectively (Keenan et al. 1984a; Doyle et al. 1985). Both the Si VIII and $\mathrm{Mg}$ IX lines are formed at temperatures around $1 \mathrm{MK}$ as can be seen from Fig. 1, where the ionic fractions of $\mathrm{Si}^{7+}$ and $\mathrm{Mg}^{8+}$ under ionization-equilibrium conditions are plotted in panel a) together with the corresponding contribution functions of the emission lines in b).

The Si VIII lines are observed in the first order of diffraction by the SUMER instrument and can be recorded with both detectors. The method is ideally suited to line-ratio measurements, because the small wavelength separation facilitates the radiometric calibration. The low radiances of the lines on the disk (cf. Wilhelm et al. 1998; Curdt et al. 2001) eliminate interference with scattered radiation in the instrument to a large extent. Furthermore, no problems are caused by blends of other spectral lines in the first or second order (cf. Curdt et al. 2004). Since the Si VIII lines are emitted from the same ion, abundance and ionic-fraction variations are also no concern. However, the ratio varies less as a function of the density in the low-density plasma of coronal holes than in the optimum range near $3 \times 10^{8} \mathrm{~cm}^{-3}$, and thus small uncertainties in the determination of the line ratios will induce much larger relative uncertainties of the resulting densities.

The close neighbourhood in wavelength of the Mg IX lines again provides a calibration advantage; the relatively low radiances of the lines on the disk lead to small contributions of scattered radiation in the instrument; and abundance variations are not a problem. Only Detector B can record spectral lines with wavelengths near $70 \mathrm{~nm}$ in the first order. In the second order,

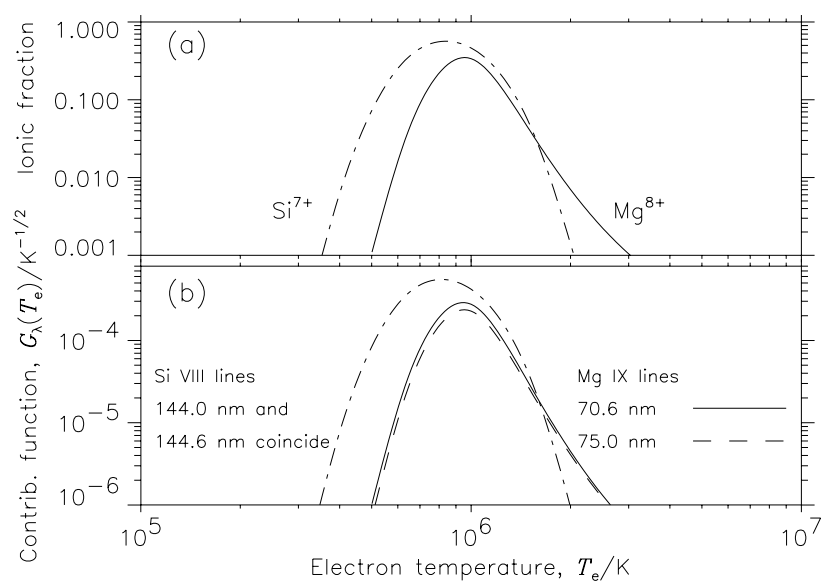

Fig. 1. a) Ionic fractions of the nitrogen-like $\mathrm{Si}^{7+}$ and beryllium-like $\mathrm{Mg}^{8+}$ ions under ionization-equilibrium conditions (Mazzotta et al. 1998); b) the corresponding contribution functions of the Si VIII and Mg IX lines near $144 \mathrm{~nm}$ and $70 \mathrm{~nm}$, respectively, as a function of the electron temperature assuming electron collision excitation from the ground state (cf. Pottasch 1963; Wilhelm et al. 2004, and references therein).

these lines can also be observed with Detector A, but this option has not been employed very often. As Detector A was mostly used in the first years of the SOHO mission, line-ratio measurements of the Mg IX pair are scarce from that period.

The few observations available with Detector B were, moreover, not acquired simultaneously with the corresponding electron density measurements (Wilhelm et al. 1998). The reason for this deficiency lies in the instrumental design, which requires rapid movements of the spectrometer scan and focus mechanisms over most of their operating ranges to cover both the Si VIII and the Mg IX lines in the first order. Life-time considerations and a prudent use of the resources available precluded such fast and extended scans in the early mission phase, but, after nearly ten years of successful operation, such extreme care is no longer necessary, and the Si VIII and Mg IX line pairs have now both been recorded with Detector B in the first order for the same coronal structures almost simultaneously.

Another concern was the azimuthal (east-west) scan mechanism of the telescope. From the end of 1996 onwards, the mechanism failed with the nominal current applied to the driving motor due to high friction of the lead screw, but functioned in a high-current mode. Nevertheless, it was used only for pointing purposes and not for raster-step images for quite some time. With such a restriction, the SUMER slit, normally aligned in the north-south direction, had to be pointed either to a plume or a lane in the polar regions of the Sun. Pointing uncertainties sometimes made it difficult to determine how well the desired target was hit, which is particularly critical for narrow plumes and lanes (Doschek et al. 1997; Wilhelm et al. 1998). Alternatively, a scheme was employed that relied on a configuration in which the SUMER slit was orientated in the east-west direction. Plume and inter-plume crossings of the slit at a certain height above the limb could be defined more easily in such a manner (Wilhelm \& Bodmer 1998; Gabriel et al. 2003), but one of the infrequent $90^{\circ} \mathrm{SOHO}$ roll manœuvres was required to implement these observations. As for the spectrometer mechanisms, life-time considerations are now no longer of primary importance for the azimuthal drive. The critical devices at this stage are the detectors, and it is imperative to optimize their utilization. 


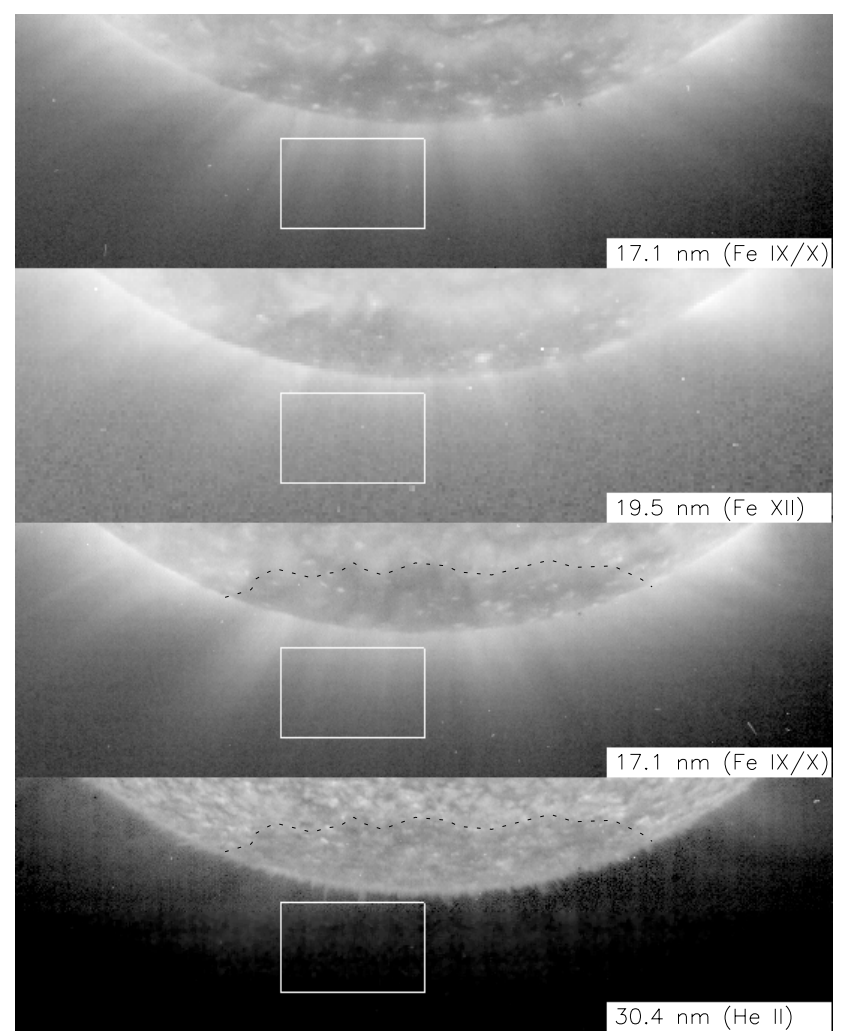

Fig. 2. Images of the south polar region of the Sun in the EIT windows with wavelengths around $17.1 \mathrm{~nm}(\mathrm{Fe}$ IX/X), $19.5 \mathrm{~nm}$ (Fe XII) and $30.4 \mathrm{~nm}$ (He II) (courtesy of the EIT consortium). The image on the top was taken at 19:00 UTC on 23 May 2005, and the one in $19.5 \mathrm{~nm}$ at 23:24 UTC just before the SUMER raster scan treated in this report was initiated. After the scan, the second Fe IX/X image is from 19:00, and the He II image from 19:19 UTC. The area studied is marked in all panels. The boundary of the coronal hole is traced in the He II image and repeated in the Fe IX/X image of 24 May.

Analysis of the Si VIII measurements in Sect. 4 first follows Gabriel et al. (2003) in assuming that the fraction occupied by plume material along the LOS is a free parameter. In order to simulate the results of the observations, the plume electron density divided by that of the inter-plume region is considered as another free parameter. In this simulation, the electron density of plumes as a function of height and estimates of the electron temperatures in plumes and inter-plume regions are taken from earlier investigations. The data are secondly treated without any assumptions on the electron density and temperatures.

\section{Observations in May 2005}

The observations to be presented were made on 24 May 2005 during the fifteenth SOHO-MEDOC ${ }^{1}$ campaign $^{2}$. The angular solar radius was $R_{\odot}=957^{\prime \prime}$, at that time, with a scale of $727 \mathrm{~km}$ per second of arc. A well-developed coronal hole was located near the south pole of the Sun. Its northernmost latitude was approximately $-68^{\circ}$. In Fig. 2 images of this

\footnotetext{
${ }^{1}$ Multi-Experiment Data Operations Center for SOHO at Orsay, France.

2 The raw SUMER data are in the public domain and can be obtained from http://idc-medoc.ias.u-psud.fr/index.jsp, http://sohodata.nascom.nasa.gov/cgi-bin/gui or http://www.mps.mpg.de/projects/soho/sumer/FILE/ SumerEntryPage.html
}

region taken by the Extreme-ultraviolet Imaging Telescope (EIT) (Delaboudinière et al. 1995) shortly before and after our observations are shown in spectral windows primarily representative of the Fe IX/X, Fe XII, and He II lines. Measurements of the size of the hole during the solar rotation encompassing our observations using the EIT images from 10 May to 6 June 2005 show that there was quite some variability with a mean angular heliocentric diameter of $\approx 44^{\circ}$. On 24 May 2005, the south pole was at a position of $1.6^{\circ}$ in heliocentric angle in front of the limb $\mathrm{b}^{3}$, and thus the boundary of the coronal hole on the far side was likely to be closer to the polar limb than on the visible side. It is therefore necessary to check that no plasma of the quiet Sun outside the coronal hole contaminated the measurements in the background. In the foreground, the situation is much more favourable. The position of the coronal-hole boundary can actually be measured there to be $\approx 28^{\circ}$ from the limb at a co-latitude of $\approx 26^{\circ}$.

Several plumes evidently extended from this polar coronal hole. The eastern section of it was selected as scan range. The centre of the $4^{\prime \prime} \times 300^{\prime \prime}$ slit (Slit No. 1) was initially pointed to $x=0^{\prime \prime}$ and $y=-1150^{\prime \prime}$ in solar angular coordinates or to $X=0 \mathrm{Mm}$ and $Y=-836 \mathrm{Mm}$. A pointing calibration in $y$ using the solar limb indicated uncertainties of less than $5^{\prime \prime}$ at 16:56 UTC on 23 May. There were three reasons for pointing relatively high above the limb: (1) the counting rate at the portion of the wide slit near the Sun should not be too high as a general safety procedure for Detector B; (2) the Mg IX lines suffer from H I Lyman continuum absorption at altitudes below $\approx 30 \mathrm{Mm}$ (Wilhelm et al. 1998); and (3) other coronal-hole observations, especially in the framework of SOHO-JOP 158, had been executed at the same position shortly before this sequence. The combined data sets might eventually provide additional insight, even into height ranges not considered in this report (cf. Sect. 3).

The slit was moved from $x=0^{\prime \prime}$ to $x=-250^{\prime \prime}$ in 20 steps of $12.5^{\prime \prime}$. At each of the 21 pointing positions of this observational sequence, six exposures with sampling times of $300 \mathrm{~s}$ were taken as follows: (1) two with a wavelength $\lambda=143.7 \mathrm{~nm}$ and reference pixel 511, covering the Si VIII lines at rest wavelengths $\lambda_{0}=144.049 \mathrm{~nm}$ and $144.576 \mathrm{~nm}$ on the potassium-bromide photocathode, as well as the instrumentally-scattered O II line at $71.85 \mathrm{~nm}$ in the second order as wavelength reference; (2) two each for the Mg IX lines at $70.606 \mathrm{~nm}$ and $74.955 \mathrm{~nm}$, both with a reference pixel of 500. Together with the Mg IX $70.6 \mathrm{~nm}$ line, the scattered O III line at $70.387 \mathrm{~nm}$ was recorded as wavelength reference. The first exposure of this run was taken at 01:17 and the last one at 12:55 UTC. The output of the full detector array of each exposure was telemetered to the ground stations.

It should be noted from the outset that this scheme is optimized for observations concerning the stationary plasma conditions and not for the detection of any wave activity and other rapid variations. These will, therefore, not be treated in this study. It is, however, of interest here that polar-plume oscillations are often interpreted in terms of compressional waves in magnetic field tubes (cf. Ofman et al. 2000; Banerjee et al. 2000b).

\section{Data evaluation}

In Figs. 3 and 4, the radiances of Si VIII $144.6 \mathrm{~nm}, 144.0 \mathrm{~nm}$ and $\mathrm{MgIX} 70.6 \mathrm{~nm}, 75.0 \mathrm{~nm}$ are displayed as they were

3 The $\mathrm{Y}_{\mathrm{ii}}$ axis of the $\mathrm{SOHO}$ inter-instrument coordinate system is in the plane containing the solar rotation axis (Domingo et al. 1995). 


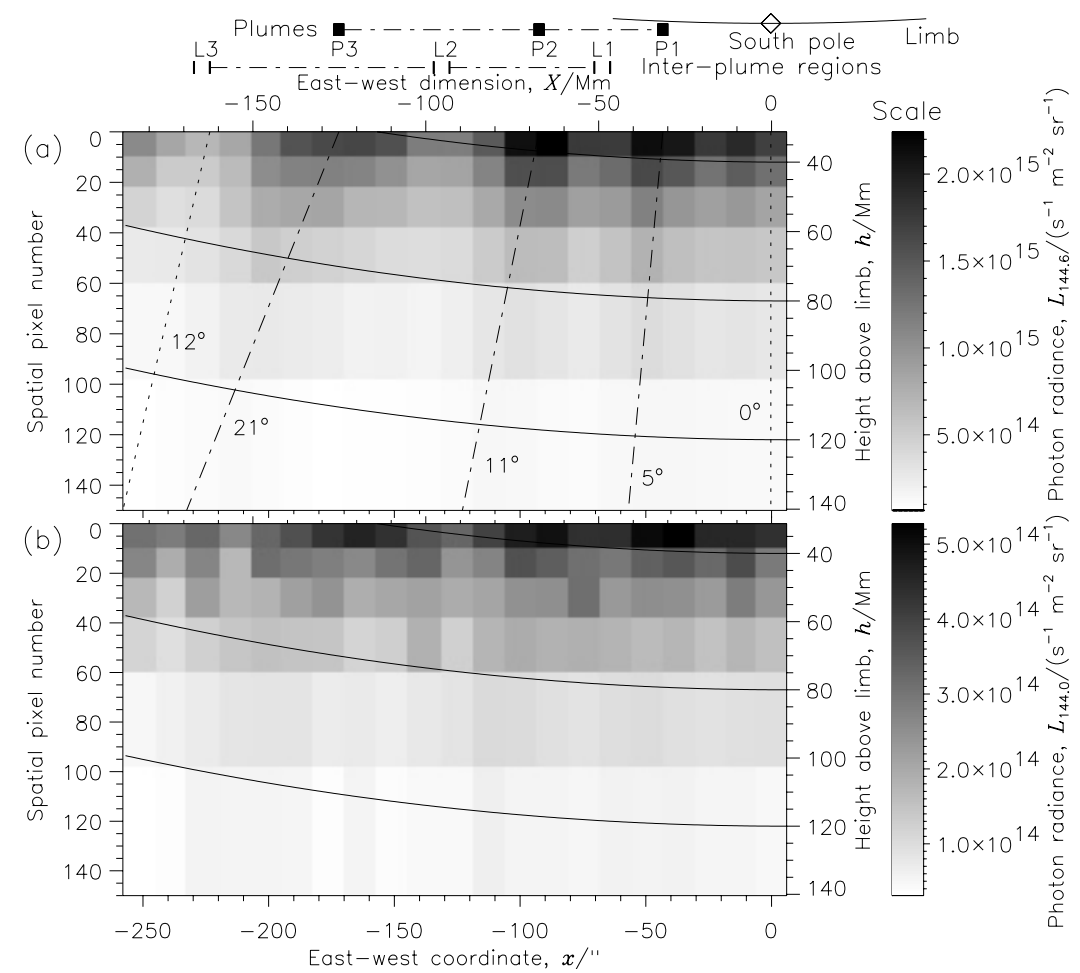

Fig. 3. Maps of the Si VIII $144.6 \mathrm{~nm}$ and $144.0 \mathrm{~nm}$ photon radiances, a) $L_{144.6}$ and b) $L_{144.0}$, in the coronal hole. The LOS projection of the south pole of the Sun is marked in the upper margin for map a). A section of the circle with a radius of $1 R_{\odot}$ through the pole projection approximates the solar limb. The maps extend in height from $31 \mathrm{Mm}$ to $140 \mathrm{Mm}$ above the pole projection, and reach from there $182 \mathrm{Mm}$ to the east. The scales of these "negative" displays are shown on the right. Concentric circles every $40 \mathrm{Mm}$ define the closest approach of the LOS to the limb at the scan positions. Bright structures (P1, P2, P3) and dark lanes (L1, L2, L3) have been identified in the upper margin at their central positions in spatial pixel number 0 . A weaker structure and the accompanying lanes are visible between $X=0$ and $-20 \mathrm{Mm}$. In panel a), the radial directions at southern co-latitudes of $0^{\circ}$ and $12^{\circ}$ are shown as dotted lines, and the plume directions $\left(5^{\circ}, 11^{\circ}, 21^{\circ}\right)$ expected after the magnetic field model of Banaszkiewicz et al. (1998) are indicated near the ridges of P1, P2, P3.

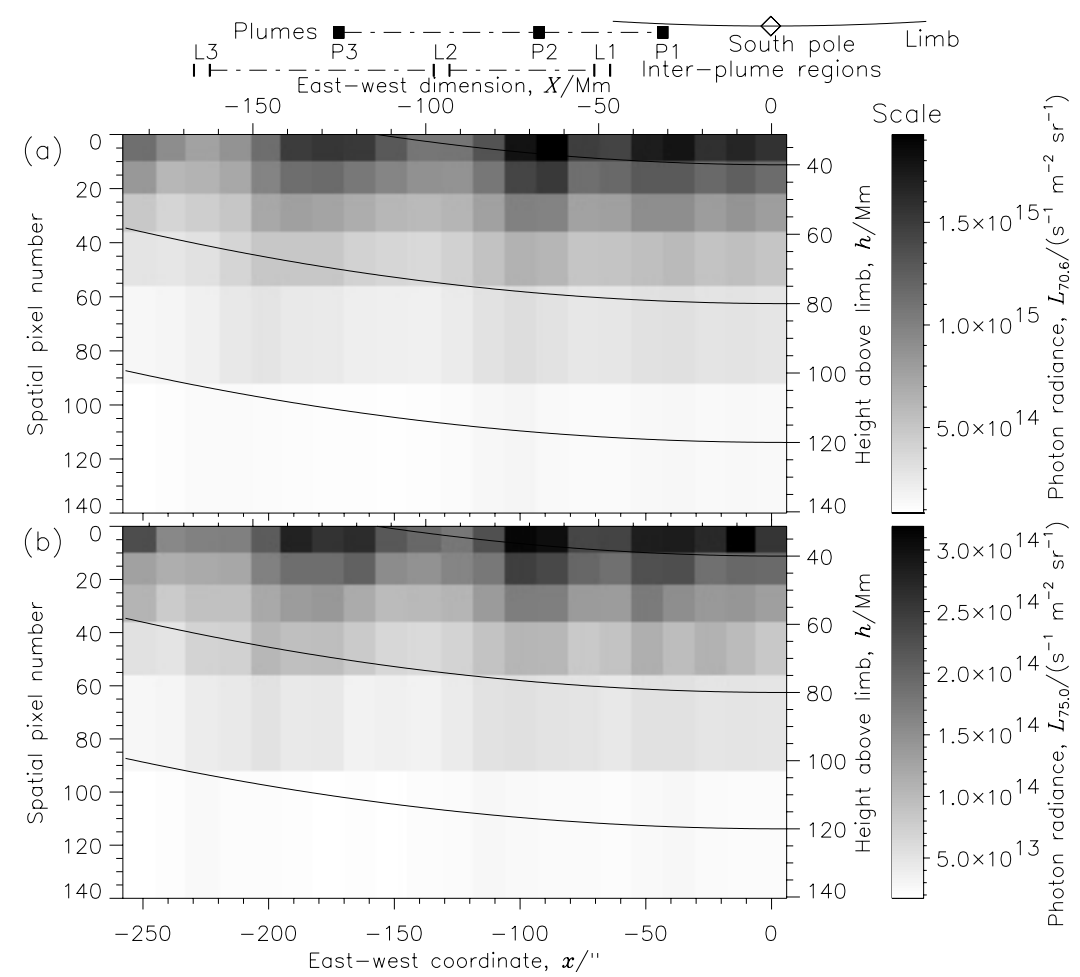

Fig. 4. Maps of the Mg IX $70.6 \mathrm{~nm}$ and the $75.0 \mathrm{~nm}$ photon radiances, a) $L_{70.6}$ and b) $L_{75.0}$, in a format similar to that of Fig. 3. In comparison to the $\mathrm{Si}$ VIII lines, there is a slight shift in the spatial pixel numbers with respect to the height scale, due to the wavelength dependence of the instrumental magnification factor. 
Table 1. The linear correlation coefficients between the radiances of the Si VIII and $\mathrm{Mg}$ IX lines together with the $3 \sigma$ confidence levels.

\begin{tabular}{ll|cccc}
\hline \hline & & \multicolumn{4}{|c}{ Correlation coefficient $^{a}, r$} \\
& & $L_{144.6}$ & $L_{144.0}$ & $L_{70.6}$ & $L_{75.0}$ \\
\hline \multirow{3}{*}{ Confidence } & $L_{144.6}$ & 1 & 0.970 & 0.992 & 0.982 \\
level $^{b}, 3 \sigma$ & $L_{74.0}$ & 0.016 & 1 & 0.977 & 0.973 \\
& $L_{70.6}$ & 0.004 & 0.012 & 1 & 0.992 \\
& $L_{75.0}$ & 0.009 & 0.014 & 0.004 & 1 \\
\hline
\end{tabular}

${ }^{a}$ In the upper right-hand section (and on the diagonal); ${ }^{b}$ in the lower left-hand section.

obtained from the measured line profiles ${ }^{4}$. The diagrams clearly show several distinct high and low radiance regions that are labelled in the upper margin of the diagrams with P1 to P3 or L1 to L3. The variations across the scan area are considered to stem primarily from spatial structures and not from temporal fluctuations. The apparent widths of the structures in the east-west direction are $20 \mathrm{Mm}$ to $40 \mathrm{Mm}$. The correlation coefficients, $r$, between the data fields of these diagrams and the confidence levels, $3 \sigma$, are listed in Table 1 . If $|r| \geq 3 \sigma$, there is only a relative chance of $\leq 0.3 \%$ that the result is obtained by coincidence (e.g., Bronstein \& Semendjajew 1985). In parallel, Gaussian fits were produced and visually compared with the measured profiles. The Gaussian fits were also used to find the Doppler widths, $\Delta \lambda_{\mathrm{D}}$, of the Si VIII $144.6 \mathrm{~nm}$ and $\mathrm{Mg}$ IX $70.6 \mathrm{~nm}$ profiles, as well as their shifts with respect to the reference lines given in Sect. 2.

No recent flatfield of the detector array was available for these calculations. To find out whether an old flatfield of 1999 could be employed for an improvement, the correlation coefficients, $r_{\mathrm{FF}}$, between Figs. 3a and $4 \mathrm{a}$ were also calculated for data sets with flatfield applications. The coefficient $r_{\mathrm{FF}}\left(L_{144.6}, L_{70.6}\right)$ is 0.988 , and the value $r\left(L_{144.6}, L_{70.6}\right)$ without correction from Table 1 is 0.992 . Tests with other combinations of lines corroborated this result. Since the old flatfield had a slightly adverse effect, this correction was not applied. The low counting rates in the coronal hole, especially in the dark lanes, require substantial spatial binning of the detector pixels along the slit image, and thus the missing flatfield is not a serious drawback.

In order to cope with the odd-even pattern of the detector responsivity, which is caused by a non-linearity of the analog-todigital converter, binning was employed with an even pixel number in the spatial dimension. This was done in such a way that the decrease in the useful signal with height was to some extent compensated for by more binning at the expense of the spatial resolution, and, at the same time, the spatial pixels near $144 \mathrm{~nm}$ and $70 \mathrm{~nm}$ were co-aligned as much as possible. The bin sizes used are 10, 12, 16, 22, 38, 52 pixels for Si VIII, and 10, 12, 14, 20, 36, 48 pixels for Mg IX. Together with the 21 exposure positions, $6 \times 21=126$ data fields are thus available for each line. Pixels related to greater altitudes have not been evaluated at this stage, because the counting statistics were rather poor. The scan from west to east leads to changes in altitude of the closest approach along the LOS of a spatial pixel. Therefore, the bins cover height ranges $h_{01}$ to $h_{56}$ that are wider than the dimensions corresponding to the actual bin sizes (cf. Figs. 3, 4 and $6 \mathrm{~b})^{5}$.

4 The standard SUMER analysis and calibration software is available in the SolarSoft Library at

http://sohowww . nascom. nasa.gov/solarsoft/soho/sumer/.

5 An allocation of pixels to certain height intervals could improve the resolution, but would require complicated algorithms. In view of the large bin sizes, such a procedure does not seem to be justified.
The number of counts per bin during a total sampling time of two times $300 \mathrm{~s}$ for the Si VIII $144.6 \mathrm{~nm}$ line varied between 700 and 1400 in the minima and 1100 and 4400 in the maxima. From the counting statistics, we can thus expect a relative standard uncertainty of $\approx 3 \%$ for the minima and, for the maxima, values between $2 \%$ and $3 \%$. The figures for the $144.0 \mathrm{~nm}$ line are $\approx 400$ at the minima and up to 1000 for the maxima, or 5\% and $3 \%$. The ratios of these lines therefore have estimated relative uncertainties of $8 \%$ for the minima and $\approx 5 \%$ for the maxima. The evaluation of the $\mathrm{Mg}$ IX line ratio gives relative standard uncertainties of $8 \%$ and $4 \%$, respectively.

The line ratios and their uncertainties were transferred into the electron density and temperature regimes using data from Doschek et al. (1997) for Si VIII and from ADAS ${ }^{6}$ (Summers et al. 1996) for $\mathrm{Mg}$ IX. The relative uncertainty of the density is $7 \%$ for a dense plasma of $4 \times 10^{7} \mathrm{~cm}^{-3}$, whereas a relative uncertainty of $\approx 20 \%$ has to be expected at $7 \times 10^{6} \mathrm{~cm}^{-3}$. The Mg IX ratios lead to relative uncertainties in temperature of $6 \%$ and $13 \%$ in bright and dark regions, respectively. The high uncertainties are to a large extent caused by the poor counting statistics of the weak $144.0 \mathrm{~nm}$ and $75.0 \mathrm{~nm}$ lines. Ways to improve the situation are discussed later.

\section{Analysis}

An important question is whether plumes and lanes in the coronal hole have indeed been observed or instead structures related to the closed field-line regions near the edge of the coronal hole. This boundary is located at about $68^{\circ}$ southern latitude during our observations. According to the model of Banaszkiewicz et al. $(1998)^{7}$, the magnetic field vector forms an angle of $\approx 28^{\circ}$ with the vertical at a co-latitude of $22^{\circ}$, as a consequence of the superradial expansion. The LOS in the range $h_{56}$ would exit the coronal hole at a height of $\approx 210 \mathrm{Mm}$. This is shown in Fig. 5 in a schematic cross-section of a polar coronal hole. The heights $\bar{h}_{1}$ and $\bar{h}_{6}$, mean values of the ranges $h_{01}$ and $h_{56}$, are indicated as well as the LOS at an intermediate height, $h$, both for a viewing direction tangentially to the south pole and the actual one. The small difference is neglected here. The length of the LOS within the coronal hole is $z$ with $z^{\mathrm{P}}$ in plumes and $z^{\mathrm{L}}$ in lanes, i.e.

$z=z^{\mathrm{P}}+z^{\mathrm{L}}$.

From Fig. 5 it follows that

$z_{0}=2 R_{\odot} \sin 22^{\circ}=522 \mathrm{Mm}$,

$h_{0}=R_{\odot}\left(\cos 22^{\circ}-1\right)=-51 \mathrm{Mm}$

and

$z(h) \approx z_{0}+2\left(h-h_{0}\right) \tan 50^{\circ}$.

The length, $z^{\prime}$, of the LOS through the coronal hole off the central meridian at $X$ is given by

$z^{\prime} / 2=\sqrt{(z / 2)^{2}-X^{2}}$

For $X_{\max }=182 \mathrm{Mm}$, the decrease of $z^{\prime}$ relative to $z\left(\bar{h}_{1}\right)=$ $746 \mathrm{Mm}$ is $13 \%$ and only $8 \%$ relative to $z\left(\bar{h}_{6}\right)=942 \mathrm{Mm}$. These corrections are not applied explicitly, but can be considered as uncertainties of $z$.

\footnotetext{
6 Atomic Data and Analysis Structure.

7 The model has been designed for solar minimum conditions and a slightly larger coronal hole than in our case.
} 


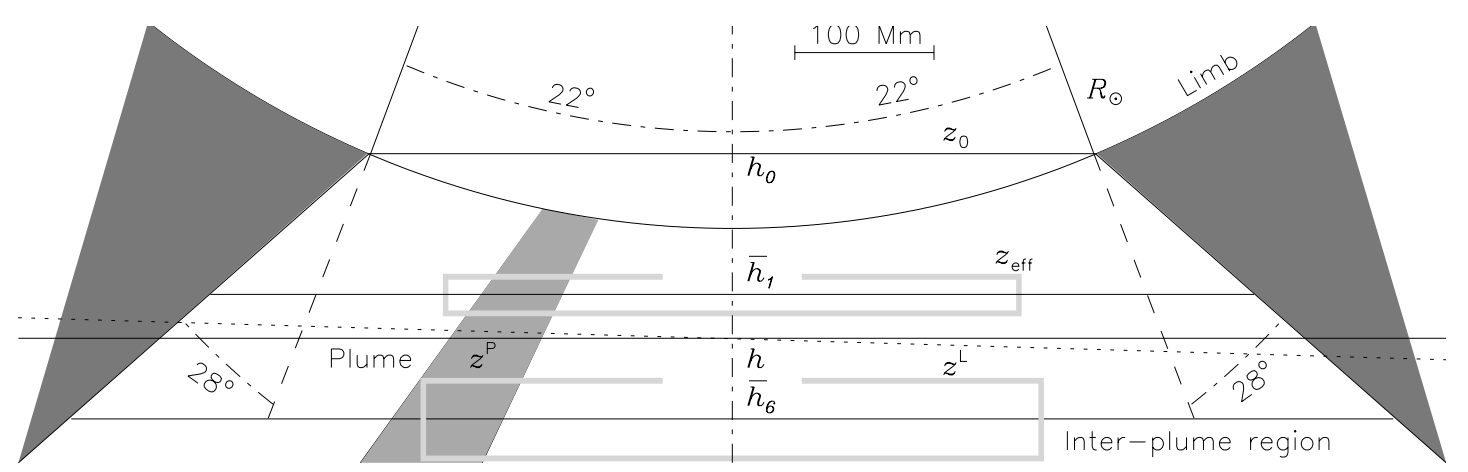

Fig. 5. Cross-section of a southern coronal hole with an angular diameter of $44^{\circ}$. The diagram is to scale and matches the solar images in Fig. 2 , but does not take the field-line bending at the coronal-hole boundaries into account. The actual LOS direction at $h$ (tilted by $1.6^{\circ}$ and rotated into the plane of the paper) is indicated as a dotted line. A sample plume is schematically shown filling a length of $z^{\mathrm{P}}$ along the LOS. In light gray are marked the "effective" emission regions of the height ranges $h_{01}$ and $h_{56}$. The space outside the coronal hole is shown in dark gray and is discussed in the text (as well as $z_{\text {eff }}, \bar{h}_{1}$, and $\bar{h}_{6}$ ).

Typical electron densities and temperatures of the quiet Sun at a height of $210 \mathrm{Mm}$ near the equator are $1.6 \times 10^{7} \mathrm{~cm}^{-3}$ and 1.3 MK (Feldman et al. 1999). At the latitudes that are relevant in this context, the values were $n_{\mathrm{e}} \leq 1.0 \times 10^{7} \mathrm{~cm}^{7}$ and $T_{\mathrm{e}} \geq 1.5 \mathrm{MK}$ in 1998 (Wilhelm 1999c; Wilhelm et al. 2002). The density is comparable to those deduced here for the lanes, but the temperature is much higher (cf. Table 3). Any loop structures in this region that might be confused with plumes would also have higher temperatures than the cool plume plasma. The data of 1998 have been re-analysed to estimate the photon radiances of the Si VIII $144.6 \mathrm{~nm}$ and Mg IX $70.6 \mathrm{~nm}$ lines along the LOS near the high-latitude boundaries of the equatorial corona. Typical values obtained are $\approx 1 \times 10^{14} \mathrm{~s}^{-1} \mathrm{~m}^{-2} \mathrm{sr}^{-1}$, which would not interfere with the diagrams of Figs. $3 \mathrm{a}$ and $4 \mathrm{a}$.

Support for our bright structures being plumes stems from the fact that they diverge super-radially. Their inclination angles with respect to the limb (cf. Fig. 3a) agree with those of the Banaszkiewicz et al. model, which was originally adjusted with the help of the wisps of ultraviolet emission at the limb. This argument hinges on the assumption that the bright structures are seen not too far from the plane of the sky. Such an assumption is reasonable as shown later. Consequently, P1, P2, P3 will be considered to be plume structures ${ }^{8}$, and L1, L2, L3 inter-plume regions.

The measurements of Fig. 3 have been displayed in two different formats in Fig. 6. The observed radiances of the Si VIII $144.0 \mathrm{~nm}$ line are plotted in both panels versus the $144.6 \mathrm{~nm}$ radiances with different plot symbols for the height ranges $h_{01}$ to $h_{56}$. A few immediate conclusions can be drawn from the distribution of the data points, if temporal variations are not of major importance: (1) the spatial variation of $L_{144.6}$ is more than a factor of three in all height ranges, whereas $L_{144.0}$ varies by less than two. With the shape of the Si VIII line ratio, $L_{144.6} / L_{144.0}$, as a function of the relevant electron density (cf. Doschek et al. 1997), this means that the density averaged over the LOS increases with the radiance; (2) within each height range, the variation of $L_{144.0}$ as a function of $L_{144.6}$ appears to be more or less linear; (3) spatial variations of the electron density that are uniform along the LOS cannot account for these observations. The radiation is therefore assumed to originate from two distinct plasmas, namely plume and inter-plume regions.

\footnotetext{
${ }^{8}$ It is by no means certain that each bright structure can be associated with a single plume. It might be an agglomeration of several plumes along the LOS.
}

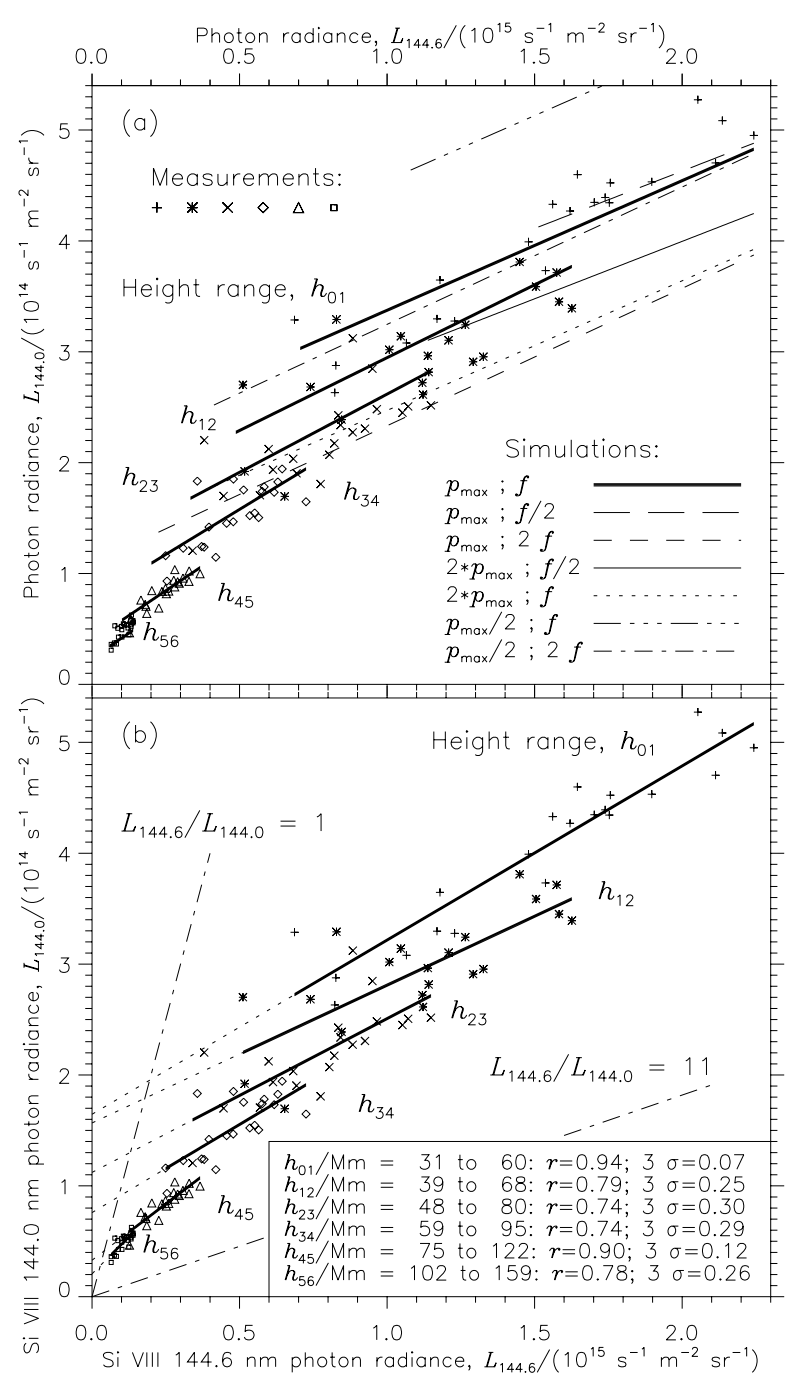

Fig. 6. The radiance of the Si VIII $144.0 \mathrm{~nm}$ line plotted as a function of the $144.6 \mathrm{~nm}$ radiance. a) Measured data points compared with simulated relations between the radiances $L_{144.6}$ and $L_{144.0}$ (cf. Table 2). Variations in the plume fraction and density ratio are shown with a factor of two up and down for the range $h_{01}$. b) Data points (repeated) and linear regression lines in each height range. The correlation coefficients, $r$, the $3 \sigma$ confidence level, and the (overlapping) height ranges, $h_{01}$ to $h_{56}$, are given in the inset. The line labelled $L_{144.6} / L_{144.0}=1$ shows the asymptotic value of the ratio reached at $n_{\mathrm{e}} \approx 1 \times 10^{6} \mathrm{~cm}^{-3}$, whereas $L_{144.6} / L_{144.0}=11$ refers to an electron density of $n_{\mathrm{e}}=1 \times 10^{8} \mathrm{~cm}^{-3}$. 
Table 2. Assumptions on the plume electron densities in the height ranges $h_{01}$ to $h_{56}$, the parameters ${ }^{a}$ of the best fit in Fig. 6a, and the resulting scale factors.

\begin{tabular}{lccc}
\hline \hline $\begin{array}{l}\text { Height } \\
\text { range }\end{array}$ & $\begin{array}{c}\text { Electron } \\
\text { density, } \\
n_{\mathrm{e}}^{\mathrm{P}} /\left(10^{6} \mathrm{~cm}^{-3}\right)\end{array}$ & $\begin{array}{c}\text { Plume } \\
\text { fraction, } \\
p_{\max }\end{array}$ & $\begin{array}{c}\text { Scale factor, } \\
z_{\mathrm{eff}} B_{144.6} / \\
\left(10^{-9} \mathrm{~m}^{4} \mathrm{~K}^{1 / 2} \mathrm{~s}^{-1} \mathrm{sr}^{-1}\right)\end{array}$ \\
\hline$h_{01}$ & 65 & 0.084 & 8.74 \\
$h_{12}$ & 57 & 0.090 & 7.85 \\
$h_{23}$ & 51 & 0.094 & 6.72 \\
$h_{34}$ & 43 & 0.100 & 5.70 \\
$h_{45}$ & 38 & 0.100 & 3.69 \\
$h_{56}$ & 33 & 0.050 & 2.21 \\
\hline
\end{tabular}

${ }^{a}$ The density ratio $f=5.3$ provided the best fits for all heights.

Under this assumption, several simulations are superimposed on the data points in Fig. 6a. The contributions were calculated with the help of

$L_{\lambda}=L_{\lambda}^{\mathrm{P}}+L_{\lambda}^{\mathrm{L}} ; \lambda=144.6 \mathrm{~nm}$ and $144.0 \mathrm{~nm}$

and

$L_{144.6}^{\mathrm{P}}=z_{\text {eff }} B_{144.6} G_{144.6}\left(T_{\mathrm{e}}^{\mathrm{P}}\right)\left(n_{\mathrm{e}}^{\mathrm{P}}\right)^{2} p$,

$L_{144.6}^{\mathrm{L}}=z_{\text {eff }} B_{144.6} G_{144.6}\left(T_{\mathrm{e}}^{\mathrm{L}}\right)\left(n_{\mathrm{e}}^{\mathrm{P}} / f\right)^{2}(1-p)$,

$L_{144.0}^{\mathrm{P}}=L_{144.6}^{\mathrm{P}} / R^{\mathrm{P}}$,

$L_{144.0}^{\mathrm{L}}=L_{144.6}^{\mathrm{L}} / R^{\mathrm{L}}$,

where $B_{144.6}$ is a scale factor ${ }^{9}$ that, together with $z_{\text {eff }}$ (later identified with the effective length of the LOS in the plasma), are used to adjust the maximum of the simulation in each height range to the highest measured radiance, $L_{144.6}^{\max } ; G_{\lambda}\left(T_{\mathrm{e}}\right)$ is the contribution function of Fig. 1 specified for the Si VIII $144.6 \mathrm{~nm}$ line with $T_{\mathrm{e}}^{\mathrm{L}}=8.7 \times 10^{5} \mathrm{~K}$ and $T_{\mathrm{e}}^{\mathrm{P}}=7.3 \times 10^{5} \mathrm{~K}$. These estimates ${ }^{10}$ are taken from Wilhelm et al. (1998), along with the plume densities, $n_{\mathrm{e}}^{\mathrm{P}}$. The electron density in the inter-plume regions is obtained from $n_{\mathrm{e}}^{\mathrm{L}}=n_{\mathrm{e}}^{\mathrm{P}} / f$ with a density ratio, $f$, between plumes and their environment. The fraction of plume plasma along the LOS, $p$, varies between 0 and $p_{\max }$. The line ratios, $R=L_{144.6} / L_{144.0}$, follow directly from the densities, $n_{\mathrm{e}}^{\mathrm{P}}$ and $n_{\mathrm{e}}^{\mathrm{L}}$. The values of $f$ and $p_{\max }$ were adjusted to find the best representation. The corresponding parameters are listed in Table 2. Some variations ${ }^{11}$ were also performed to demonstrate the sensitivity of the method. A factor of two increase or decrease of $p_{\max }$ and/or $f$ led to a total mismatch.

A second method of analysing the Si VIII observations is shown in Fig. 6b. The linear relation between the radiances of $L_{144.0}$ and $L_{144.6}$ in each height range (with some scatter of the data points that can quantitatively be understood as caused by the uncertainties of the weak line) can be described by correlation coefficients, $r$, between the line radiances. Figure 7 shows the corresponding plots for the Mg IX $75.0 \mathrm{~nm}$ and $70.6 \mathrm{~nm}$ lines with a behaviour similar to that of the Si VIII lines, albeit in a compressed form.

9 This factor is predominantly $(\approx 2 / 3)$ dependent on the plume data. A difference in the plume or inter-plume regimes, if present, cannot be deduced at this stage.

10 With these electron temperatures close to the broad maximum of the Si VIII contribution function, $G_{144.6}\left(T_{\mathrm{e}}\right)$ is nearly constant and has little influence on the results.

11 Only included for the height range $h_{01}$ in Fig. 6a.

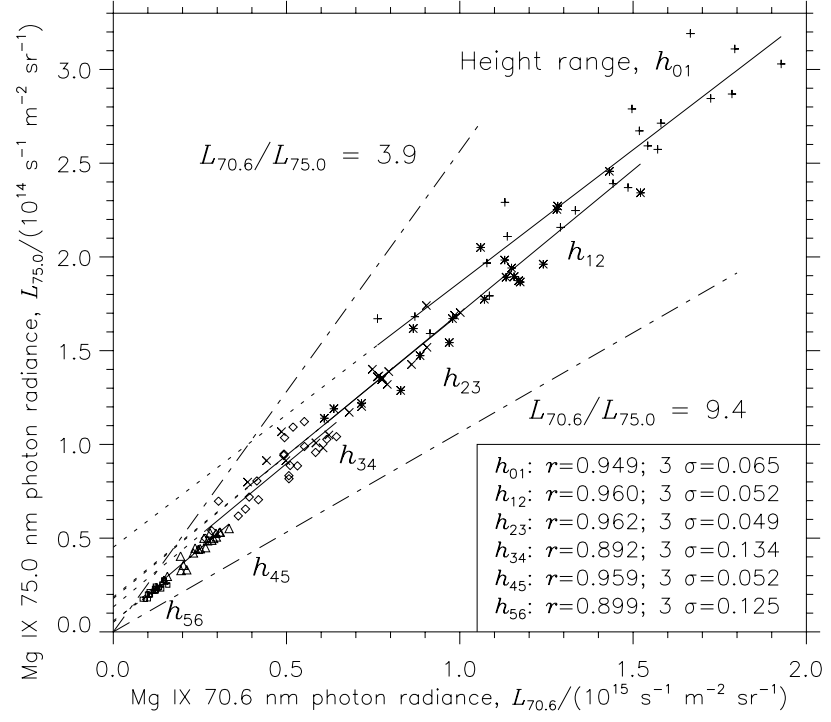

Fig. 7. The radiance of the $\mathrm{Mg} I X 75.0 \mathrm{~nm}$ line as a function of the $70.6 \mathrm{~nm}$ radiance in the same height ranges as in Fig. 6b. The correlation data are given in the inset. The lines labelled $L_{70.6} / L_{75.0}=9.4$ and 3.9 correspond to $T_{\mathrm{e}} \approx 5 \times 10^{5} \mathrm{~K}$ and $2 \mathrm{MK}$, respectively.

The correlations in Figs. $6 \mathrm{~b}$ and 7 are so close (cf. the $r$ and $\sigma$ values in the insets) that it can be assumed that the relationships provide a reliable interpolation scheme for the weaker lines at $144.0 \mathrm{~nm}$ and $75.0 \mathrm{~nm}$. This improves the uncertainties of the weak lines, bringing them close to the levels of the strong lines. Given this advantage, the $144.0 \mathrm{~nm}$ and $75.0 \mathrm{~nm}$ radiances are taken from the fits in Figs. 6b and 7 in the following discussion, unless the use of uncorrected data is explicitly stated.

The need to bin even pixel numbers leads to a minor complication in spatially adjusting both the Mg IX and the Si VIII data ${ }^{12}$. It was therefore assumed that the radiance ratios at all positions in the east-west direction should be monotonic functions of the height, which could be justified by the fact that most of the uncorrected ratios could be approximated by linear fits between $\bar{h}_{1}$ and $\bar{h}_{6}$ with high confidence (typical values: $r \approx 0.8$ and $3 \sigma \approx 0.3$ ). Relative adjustments of $+5 \%$ and $-6 \%$ for $\mathrm{Mg}$ IX and $+25 \%$ and $-11 \%$ for Si VIII had to be made. In a subsequent step, the Si VIII $144.0 \mathrm{~nm}$ and the Mg IX $75.0 \mathrm{~nm}$ radiances were rescaled in order to be consistent with the ratio adjustments.

The adjusted Si VIII ratios were converted into electron densities and are displayed in Fig. 8a, and the electron temperatures obtained from the adjusted Mg IX ratios are shown in Fig. 8b. This gives only a rough idea of these quantities, because they are derived from radiances averaged along the LOS. Together with Figs. 3 and 4, it is nevertheless obvious that the brighter regions are caused by denser and cooler plasmas than the darker ones. A quantitative comparison of these density and temperature distributions in the scan area gives a linear correlation coefficient $r\left(n_{\mathrm{e}}, T_{\mathrm{e}}\right)=-0.813$ with a $3 \sigma$ confidence level of 0.091 . Even the original density and temperature maps ${ }^{13}$ are significantly anticorrelated $(r=-0.34 ; 3 \sigma=0.23)$.

In line with the outcome of the forward calculation (cf. Table 2), the plume fraction along the LOS shown in Fig. 5 is rather small. Could curtain-like structures crossed obliquely by the LOS be an explanation? To investigate this question further,

\footnotetext{
12 The radiances are steeply decreasing with height. Small spatial offsets between members of line pairs thus affect their ratio.

13 Using values without interpolation and ratio adjustment.
} 


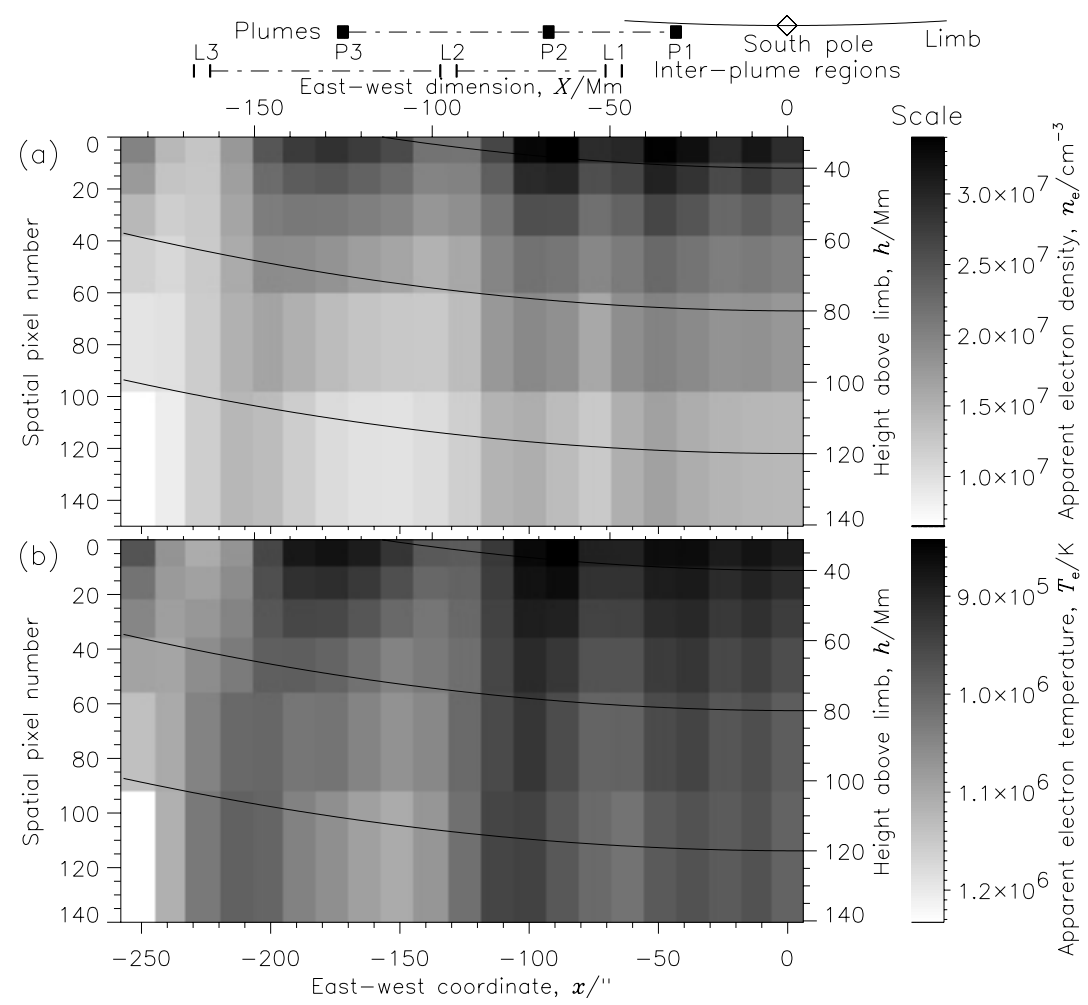

Fig. 8. a) Map of the electron density deduced from the Si VIII radiances averaged along the LOS. b) The corresponding map of the electron temperature determined from the Mg IX line pair.

Table 3. Electron densities and temperatures in the height ranges $h_{01}$ to $h_{56}$ together with the LOS lengths.

\begin{tabular}{|c|c|c|c|c|c|c|c|c|}
\hline \multirow{2}{*}{$\begin{array}{l}\text { Height } \\
\text { range }\end{array}$} & \multicolumn{2}{|c|}{ Mean height, } & \multicolumn{2}{|c|}{ Length of LOS, } & \multicolumn{2}{|c|}{ Electron number density, $n_{\mathrm{e}} /\left(10^{6} \mathrm{~cm}^{-3}\right)$} & \multicolumn{2}{|c|}{ Electron temperature, $T_{\mathrm{e}} / \mathrm{MK}$} \\
\hline & $\bar{h} / \mathrm{Mm}$ & $\bar{h} / R_{\odot}$ & $z / \mathrm{Mm}$ & $z_{\mathrm{eff}} / \mathrm{Mm}$ & $\operatorname{Lane}^{a}\left(n_{\mathrm{e}}^{\mathrm{L}}\right)$ & Plume $\left(n_{\mathrm{e}}^{\mathrm{P}}\right)$ & Lane $\left(T_{\mathrm{e}}^{\mathrm{L}}\right)$ & Plume $\left(T_{\mathrm{e}}^{\mathrm{P}}\right)$ \\
\hline$h_{01}$ & 45 & 0.065 & 750 & 415 & 12.9 & 68.8 & 1.10 & 0.73 \\
\hline$h_{12}$ & 53 & 0.076 & 768 & 419 & 12.5 & 53.8 & 1.10 & 0.76 \\
\hline$h_{23}$ & 64 & 0.092 & 796 & 423 & 10.7 & 45.0 & 1.12 & 0.77 \\
\hline$h_{34}$ & 77 & 0.111 & 826 & 428 & 10.7 & 40.7 & 1.12 & 0.78 \\
\hline$h_{45}$ & 98 & 0.141 & 876 & 436 & 9.35 & 33.6 & 1.15 & 0.77 \\
\hline$h_{56}$ & 130 & 0.186 & 952 & 447 & 7.84 & 34.2 & 1.17 & 0.69 \\
\hline
\end{tabular}

${ }^{a}$ Formally, the density values of the lanes must be considered as upper limits, should the assumption that there was a "plume-free" region not be met.

the $L_{144.6}$ and the adjusted radiances, $L_{144.0}^{*}$, are shown in Fig. 9 at low levels on a larger scale than in Fig. $6 \mathrm{~b}$. If the raster scan has encountered pure lane conditions somewhere, $L_{144.6}^{\min } / L_{144.0}^{* \min }$ is representative of the inter-plume density at the corresponding altitude (defined by the inverse slope of the broken line). The resulting densities, $n_{\mathrm{e}}^{\mathrm{L}}$, are listed in Table 3 .

The allocation of the measurements to a certain height poses a difficulty, because the height and the electron density vary along an LOS. Weighted mean emission heights, $\bar{h}_{\text {eff }}$, of the radiation have therefore been determined by assuming $L \propto n_{\mathrm{e}}^{2}$ and that the lane densities, $n_{\mathrm{e}}^{\mathrm{L}}$, can be applied and exponentially extrapolated to greater heights. It is found that the $\bar{h}_{\text {eff }}$ are greater than the $\bar{h}$ in Table 3 by $\approx 17 \mathrm{Mm}$. Along the same lines, effective lengths, $z_{\mathrm{eff}}$, are obtained by considering the diminishing contributions to the radiances of LOS sections in the fore- and background at greater heights and thus with lower electron densities. Compared to $z$, the $z_{\text {eff }}$ values are rather uncertain and relatively small, limiting the main radiance contributions to the centre of the coronal hole. Indirectly, this improves the useful height resolution of our LOS observations. This fact can also be seen as an argument against major effects from outside the coronal hole (cf. Fig. 5). An LOS length $z^{*}=2 z$ with an electron density outside the coronal hole that is a factor of two higher than inside would lead to a relative increase of $z_{\text {eff }}$ of less than $13 \%$.

Since the plumes occupy only a small fraction of the LOS, the term $(1-p)$ in Eq. (4) can be neglected in a first approximation, and the radiances $L_{144.6}^{\mathrm{L}}=L_{144.6}^{\min }$ and $L_{144.0}^{* \mathrm{~L}}=L_{144.0}^{* \min }$ can be subtracted from the $L_{144.6}$ and $L_{144.0}^{*}$ values to get estimates of the plume contributions. From $L_{144.6}^{\mathrm{P}} / L_{144.0}^{* \mathrm{P}}$, the plume electron densities, $n_{\mathrm{e}}^{\mathrm{P}}$, which are constant in the various height ranges, can then be deduced as given in Table 3. They are compatible with earlier data obtained with the help of the Si VIII ratio (Doschek et al. 1997) and with the assumptions in Table 2. The inter-plume densities are very low, approximately a factor of five lower than in plumes (confirming the forward calculation). They are in excellent agreement with white-light measurements in voids of coronal holes during eclipses (Koutchmy 1994) and with Si VIII line-ratio observations in very dark regions of coronal holes (Wilhelm 1999a). The plume and inter-plume density ratios also agree with eclipse observations in 1998 (Lites et al. 1999). The scale heights in plumes and lanes are $H \approx 110 \mathrm{Mm}$ 


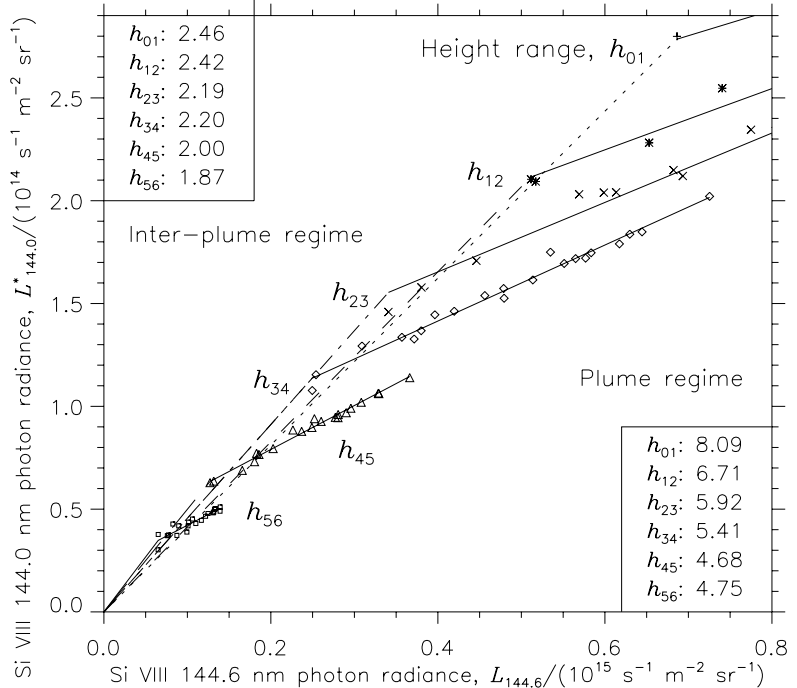

Fig. 9. The radiance $L_{144.0}^{*}$ as a function of the Si VIII $144.6 \mathrm{~nm}$ radiance. The format of the diagram is similar to that of Fig. 6b, but only the portion of the low radiances is shown. The inverse slopes of the plume regime are given in the lower inset. For the inter-plume region, the values $R=L_{144.6}^{\min } / L_{144.0}^{* \min }$ are tabulated in the upper inset.

and $160 \mathrm{Mm}$, respectively. Under hydrostatic equilibrium conditions in a fully ionized plasma with a helium fraction of $5 \%$, the corresponding temperatures would be $T_{\mathrm{S}} \approx 1.2 \mathrm{MK}$ and $2.4 \mathrm{MK}$.

The constant density of the plume plasma at a certain height means that the variations of the radiance in the solar east-west direction can only be caused by changes in the amount of plume material along the LOS. Taken together with the small fraction $p_{\max } \leq 0.1$, this result is not compatible with a curtain-like plume structures having large depth-to-width ratios in our field of view. This conclusion implies that the direct conversion of the $L_{144.6} / L_{144.0}$ ratio into the electron densities of Fig. 8a leads to a considerable underestimation of the plume densities.

In analogy to the procedure developed for the density, a portion of Fig. 7 covering the low-radiance regime is shown enlarged in Fig. 10 with adjusted Mg IX $75.0 \mathrm{~nm}$ data. The inverse slopes of the solid lines then provide the electron temperatures of plumes, $T_{\mathrm{e}}^{\mathrm{P}}$, and the dotted lines give the inter-plume values, $T_{\mathrm{e}}^{\mathrm{L}}$, all of which are listed in Table 3. Considering the counting statistics alone, the density and temperature values have relative standard uncertainties of $\approx 7 \%$ and $\approx 6 \%$, respectively. Note that the non-monotonic behaviour of the slopes as a function of altitude (at $h_{56}$ ) in the plume regime in Figs. 9 and 10 is not formally in conflict with the adjustments made earlier. The slopes have been determined from all 21 east-west positions, whereas the ratios are adjusted separately at each position. However, the inversion of the density in Table 3 is well within our uncertainty margin and thus is not significant. By the same token, the temperature inversion in plumes is significant, but this indication would need further confirmation before a final conclusion can be reached.

With the help of the data in Table 3, we can now relate the radiance observations to the plasma conditions. For allowed spectral lines emitted by an optically-thin and homogeneous plasma in a certain radiation field, $I_{\lambda}$, one can expect

$L_{\lambda}=\left[B_{\lambda} G_{\lambda}\left(T_{\mathrm{e}}\right) n_{\mathrm{e}}^{2}+\Phi_{\lambda} I_{\lambda} n_{\mathrm{e}}\right] z$

where $L_{\lambda}$ is the radiance of an emission line; $G_{\lambda}$ its contribution function (cf. Fig. 1); $B_{\lambda}$ is a coefficient, which is dependent on the electron collision excitation, the elemental abundance,

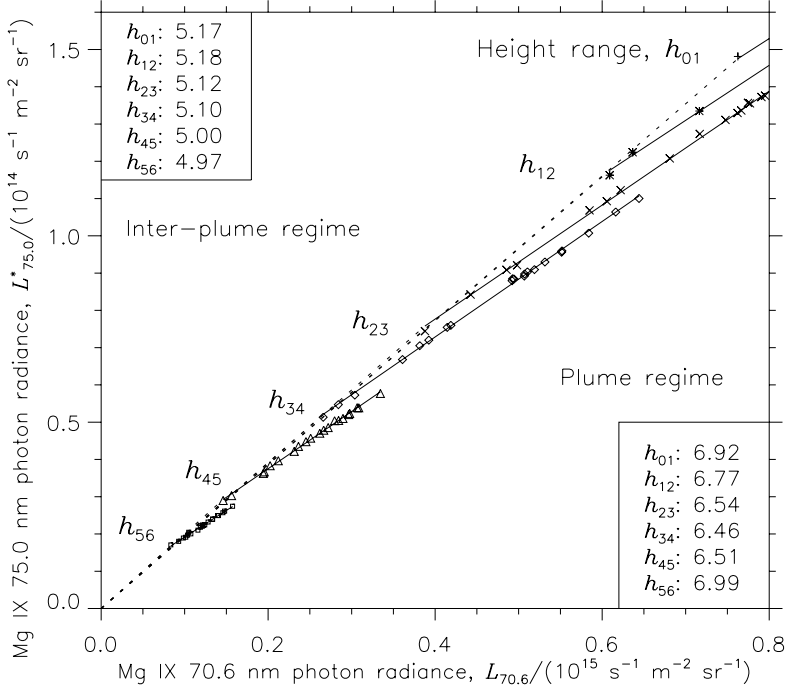

Fig. 10. The radiance $L_{75.0}^{*}$ as a function of the Mg IX $70.6 \mathrm{~nm}$ radiance. The format is similar to Fig. 9.

Table 4. Emission coefficients $^{a}$ in photon units, $B_{\lambda}^{\mathrm{L}} /\left(10^{-17} \mathrm{~m}^{3} \mathrm{~K}^{1 / 2} \mathrm{~s}^{-1} \mathrm{sr}^{-1}\right)$, in the inter-plume plasma for all height ranges and emission lines.

\begin{tabular}{lcccc}
\hline \hline Height range & \multicolumn{2}{c}{ Si VIII lines } & \multicolumn{2}{c}{ Mg IX lines } \\
& $144.6 \mathrm{~nm}$ & $144.0 \mathrm{~nm}$ & $70.6 \mathrm{~nm}$ & $75.0 \mathrm{~nm}$ \\
\hline$h_{01}$ & 3.56 & 1.45 & 5.95 & 1.15 \\
$h_{12}$ & 2.55 & 1.05 & 4.52 & 0.87 \\
$h_{23}$ & 2.52 & 1.15 & 4.31 & 0.84 \\
$h_{34}$ & 1.82 & 0.83 & 2.91 & 0.57 \\
$h_{45}$ & 1.47 & 0.74 & 2.60 & 0.52 \\
$h_{56}$ & 1.05 & 0.56 & 2.07 & 0.42 \\
\hline
\end{tabular}

${ }^{a}$ For allowed transitions and pure electron impact excitation, one would expect no height dependence in an optically-thin plasma in ionization equilibrium with only electron density and temperature variations.

etc.; $\Phi_{\lambda}$ is the corresponding coefficient related to the photoexcitation rate; and $z$ is the length of the LOS across the radiating plasma (cf., e.g., Gabriel et al. 2003; Wilhelm 2002, and references therein). Photo-excitation from the ground level will be considered to be negligible in our cases of metastable transitions (cf. Feldman et al. 1978; Keenan et al. 1984b). The emission coefficients in inter-plume regions, $B_{\lambda}^{\mathrm{L}}$, can be calculated as given in Table 4 under the assumption that, for each height range in Table 3,

$L_{\lambda}^{\min }=B_{\lambda}^{\mathrm{L}} G_{\lambda}\left(T_{\mathrm{e}}^{\mathrm{L}}\right)\left(n_{\mathrm{e}}^{\mathrm{L}}\right)^{2} z_{\mathrm{eff}}$

is valid (i.e., there is a section without any plume contribution, and the integration along the LOS is adequately taken into account by $z_{\text {eff }}$ ). The systematic decrease in $B_{\lambda}^{\mathrm{L}}$ with height could indicate LOS effects or a violation of our ionization and collisional-excitation assumptions. It is important to note that even a photo-excitation term in Eq. (6) could not improve the situation, because it would be only linearly dependent on the density.

Since the dependence of the radiance on the density, temperature, and length is explicitly taken into account in Eq. (6), it might be informative to apply the same emission coefficients in both plumes and lanes, and write with Eq. (1) adapted to the effective lengths

$L_{\lambda}=B_{\lambda}\left[G_{\lambda}\left(T_{\mathrm{e}}^{\mathrm{L}}\right)\left(n_{\mathrm{e}}^{\mathrm{L}}\right)^{2}\left(z_{\mathrm{eff}}-z_{\mathrm{eff}}^{\mathrm{P}}\right)+G_{\lambda}\left(T_{\mathrm{e}}^{\mathrm{P}}\right)\left(n_{\mathrm{e}}^{\mathrm{P}}\right)^{2} z_{\mathrm{eff}}^{\mathrm{P}}\right]$ 


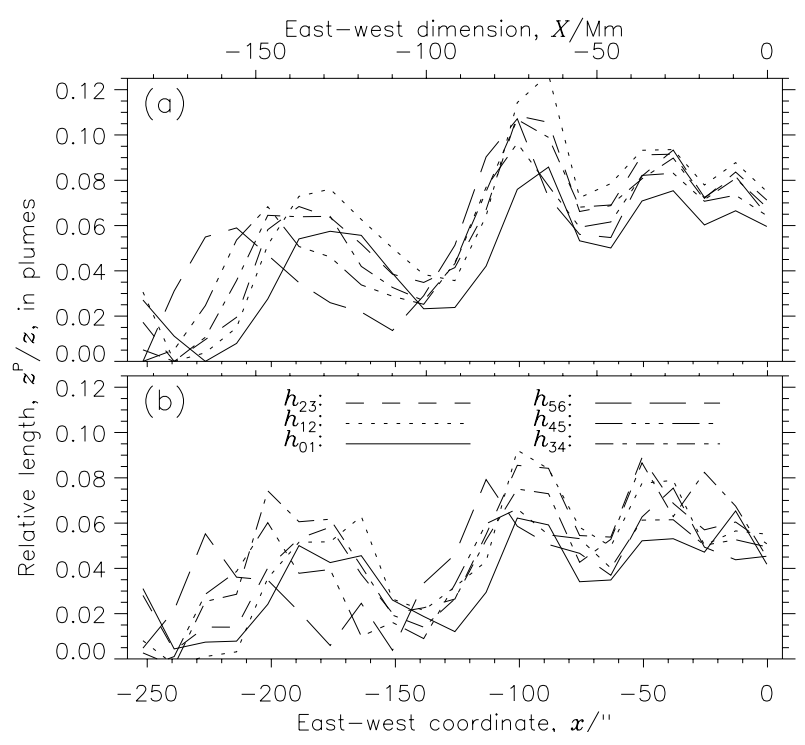

Fig. 11. The relative LOS lengths in plume plasma, $z^{\mathrm{P}} / z$, determined for the $\mathrm{Mg}$ IX lines in all six height ranges. a) $70.6 \mathrm{~nm}$; b) $75.0 \mathrm{~nm}$ (original data).

from which $z_{\text {eff }}^{\mathrm{P}}$ can be found for all data fields in Figs. 3 and 4. The results pertaining to the Mg IX lines are plotted in Fig. 11 as relative LOS lengths in plumes ${ }^{14}$. The Si VIII $144.6 \mathrm{~nm}$ line gives $z^{\mathrm{P}} / z$ values that are, in general, $33 \%$ smaller than those of the $75.0 \mathrm{~nm}$ line. The values of the $144.0 \mathrm{~nm}$ line are even a factor of four smaller. This is, of course, related to the density-sensitivity of the Si VIII lines. The Mg IX lines, on the other hand, are only weakly dependent on the electron density; the influence of the temperature, however, produces somewhat smaller maxima in the cool plumes for the $75.0 \mathrm{~nm}$ line compared to the $70.6 \mathrm{~nm}$ line. The latter has maxima of $z^{\mathrm{P}} / z$ between $8 \%$ to $12 \%$. The average $\left\langle z^{\mathrm{P}} / z\right\rangle=\left(\sum z^{\mathrm{P}} / z\right) / 21$ has also been determined for all height ranges and lines. Judged from the $70.6 \mathrm{~nm}$ line, the mean relative length along the LOS with plume material is $\left\langle z^{\mathrm{P}} \mid z\right\rangle \approx$ 0.046 in range $h_{01}$ of our raster scan ${ }^{15}$.

The spatial shift of the maxima with height in Fig. 11 agrees with the change in plume inclination. The maxima of $z^{\mathrm{P}} / z$ agree with the $p_{\max }$ values in Table 2 , and, moreover, indicate the same variation with height.

Here it must be recalled that Fig. 11 has been obtained by setting $B_{\lambda}^{\mathrm{P}}=B_{\lambda}^{\mathrm{L}}$. Although this is a plausible assumption, it might be useful to attempt a verification. A comparison of the $B_{144.6}^{\mathrm{L}}$ values in Table 4 with the scale factors in Table 2 yields $z_{\text {eff }}$ distances of $210 \mathrm{Mm}$ to $310 \mathrm{Mm}$. This discrepancy with Table 3 can be cured by assuming $B_{\lambda}^{\mathrm{P}} \approx 0.6 B_{\lambda}^{\mathrm{L}}$. Re-evaluations of Eqs. (4) and (7) under this assumption lead to relative increases of $p_{\max }$ and $z^{\mathrm{P}} / z$ by less than $60 \%$ and of $f$ by about $30 \%$, which might be considered as the relevant uncertainties.

In addition to the electron density and temperature, the effective ion temperature, $T_{\mathrm{i}, \text { eff }}$, is of interest ${ }^{16}$. It can be calculated

\footnotetext{
${ }^{14}$ Since the LOS length in plumes is deduced from the observed structures, an effective length, $z_{\text {eff }}^{\mathrm{P}}$, is obtained. Without knowing the threedimensional orientation of the plumes, a conversion to $z^{\mathrm{P}}$ is not possible. However, the scale heights in plumes and inter-plume regions are not too different, so it will be assumed that $z_{\mathrm{eff}}^{\mathrm{P}} / z_{\mathrm{eff}} \approx z^{\mathrm{P}} / z$.

15 The other lines would give smaller plume contributions.

${ }^{16}$ With only two ions observed, the separation of $T_{\mathrm{i} \text {,eff }}$ into an ion temperature, $T_{\mathrm{i}}$, and a non-thermal contribution, $\xi$, will not even be attempted here.
}

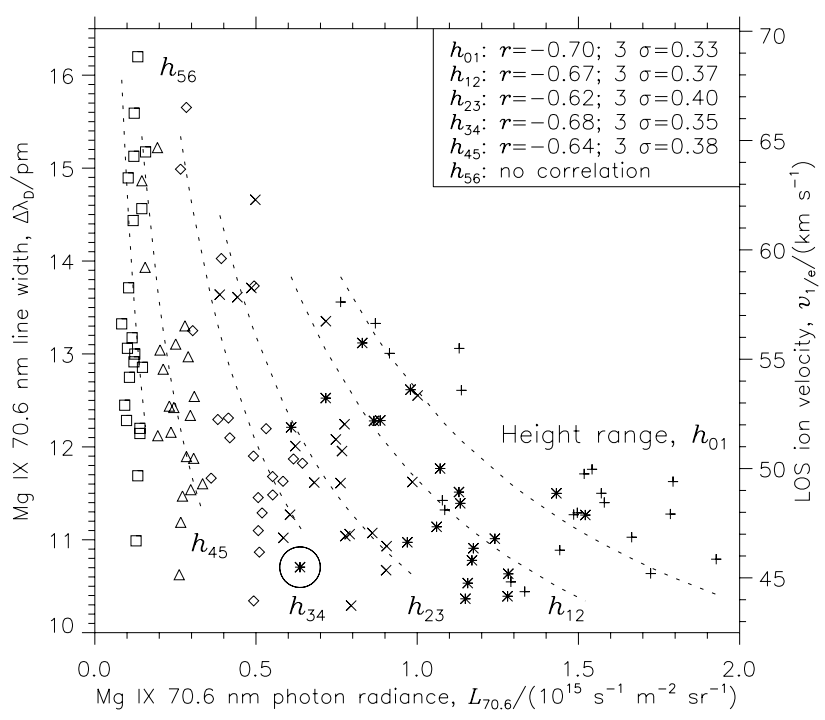

Fig. 12. The width of the Mg IX $70.6 \mathrm{~nm}$ line as a function of its radiance. The right-hand scale gives the LOS component of the unresolved turbulent ion speed. A single-Gaussian fit was adequate for all spectral profiles except for one of range $h_{12}$ that exhibited a narrow central portion and wide wings. The resulting data point turned out to be the encircled outlier. It was not taken into account in calculating the correlation coefficient. The simulated widths of the Mg IX $70.6 \mathrm{~nm}$ line at different heights are shown as dotted lines.

from the Doppler width, $\Delta \lambda_{\mathrm{D}}$, of an emission line through the most-probable LOS ion velocity of the unresolved, small-scale turbulence

$v_{1 / \mathrm{e}}=\frac{\Delta \lambda_{\mathrm{D}}}{\lambda_{0}} c_{0}$

and

$T_{\mathrm{i}, \text { eff }}=\frac{m_{\mathrm{i}}}{2 k} v_{1 / \mathrm{e}}^{2}$,

where $c_{0}$ is the speed of light in vacuum, $m_{\mathrm{i}}$ the ion mass, and $k$ the Boltzmann constant.

The measured Doppler widths are convolutions of the natural line widths with instrumental contributions. The SUMER line-width de-convolution programme was employed to obtain the corrected widths, which are displayed in Fig. 12 for the $\mathrm{Mg}$ IX $70.6 \mathrm{~nm}$ line as a function of the radiance. The LOS velocities, $v_{1 / \mathrm{e}}$, of the $\mathrm{Mg}^{8+}$ ions range from (44 to 69) $\mathrm{km} \mathrm{s}^{-1}$. Except for the highest altitudes, where the line width varies independently of the radiance, they are anticorrelated with the brightness of the emission line at all other heights. A corresponding analysis for the Si VIII $144.6 \mathrm{~nm}$ line gave similar results for $h_{01}, h_{12}$, $h_{45}$, and $h_{56}$, but no significant correlation at the $1 \sigma$ level for $h_{23}$ and $h_{34}$. The $\mathrm{Si}^{7+}$ LOS velocities ranged from (30 to 48) $\mathrm{km} \mathrm{s}^{-1}$.

A possible influence of the LOS bulk velocities in interplume regions on the line width needs to be considered. Assuming as starting point a speed of $v^{\mathrm{L}} \approx 70 \mathrm{~km} \mathrm{~s}^{-1}$ in a lane (Patsourakos \& Vial 2000) and combining it with the geometrical model in Fig. 5, extreme LOS contributions of $v_{\text {extr }}=$ $\pm v^{\mathrm{L}} \sin 50^{\circ}= \pm 54 \mathrm{~km} \mathrm{~s}^{-1}$ can be expected. Both the velocity component, $v_{\mathrm{LOS}}$, and the contributions to the radiance vary along the LOS. This can be described by a distribution function, $f\left(v_{\mathrm{LOS}}\right)$, obtained from the Banaszkiewicz et al. model and the assumption that the height dependence of $L_{70.6}$ in lanes (from the values $L_{70.6}^{\min }$ in Fig. 10) can be exponentially extrapolated to 
Table 5. The Mg IX $70.6 \mathrm{~nm}$ line-width assumptions in inter-plume regions for the simulation in Fig 12.

\begin{tabular}{lcccc}
\hline \hline $\begin{array}{l}\text { Height } \\
\text { range }\end{array}$ & $\begin{array}{c}\text { Mean } \\
\text { height, } \\
\bar{h} / \mathrm{Mm}\end{array}$ & $\begin{array}{c}\text { LOS vel- } \\
\text { ocity, } v_{1 / \mathrm{e}}^{\mathrm{L}} / \\
\left(\mathrm{km} \mathrm{s}^{-1}\right)\end{array}$ & $\begin{array}{c}\text { Line } \\
\text { width, } \\
\Delta \lambda_{\mathrm{D}} / \mathrm{pm}\end{array}$ & $\begin{array}{c}\text { effective ion } \\
\text { temperature, } \\
T_{\mathrm{i}, \mathrm{eff}} / \mathrm{MK}\end{array}$ \\
\hline$h_{01}$ & 45 & 59 & 13.9 & 5.1 \\
$h_{12}$ & 53 & 59 & 13.9 & 5.1 \\
$h_{23}$ & 64 & 62 & 14.6 & 5.7 \\
$h_{34}$ & 77 & 66 & 15.5 & 6.4 \\
$h_{45}$ & 98 & 66 & 15.5 & 6.4 \\
$h_{56}$ & 130 & 69 & 16.2 & 7.0 \\
\hline
\end{tabular}

the variations along the LOS. The line profiles observed in the dark regions can then be thought of as convolutions of the natural widths with $f\left(v_{\mathrm{LOS}}\right)$. We find relative increases in the widths between $6 \%$ and $14 \%$ as upper limits due to this effect, which will be neglected in view of the other uncertainties.

The interpretation of Figs. 9 and 10 can be a guide for understanding Fig. 12. In particular, it is critical to assume that the lowest radiance values of each height range stem from pure interplume plasma. Here the largest line widths occur. A quantitative interpretation of the narrow widths with $v_{1 / \mathrm{e}}$ close to $44 \mathrm{~km} \mathrm{~s}^{-1}$ at all altitudes and high radiance values of the $\mathrm{Mg}$ IX $70.6 \mathrm{~nm}$ line $\mathrm{e}^{17}$ is more difficult than under low-radiance conditions, because it is not immediately clear whether the value can be attributed to plumes or to a mixture of lane and plume plasmas.

By combining the geometrical results of Fig. 11 with the data in Table 3 and postulating an ad hoc value $v_{1 / \mathrm{e}}^{\mathrm{P}}=$ $35 \mathrm{~km} \mathrm{~s}^{-1}$ in plumes, it is possible to simulate the width of the Mg IX $70.6 \mathrm{~nm}$ line as a function of the radiance. The values $v_{1 / \mathrm{e}}^{\mathrm{L}}$ in Table 5 have been chosen to produce the fits in Fig. 12. To this end, Gaussian-shaped line profiles were separately determined for the plume and lane contributions in Eq. (7). The width of their sum is then the expected width ${ }^{18}$. It was overplotted as the Doppler width of a single-Gaussian fit in Fig. 12. Apart from minor differences, the characteristic features of the observations can be seen in these simulations, notwithstanding the simple method employed. It thus seems to be justified to relate the velocity of $35 \mathrm{~km} \mathrm{~s}^{-1}$ and the corresponding effective ion temperature of $1.8 \mathrm{MK}$ to the plume plasma. As for the electron temperature, there is consequently no major change in the ion temperature within our altitude range in plumes.

Finally, the Doppler shifts of the Si VIII $144.6 \mathrm{~nm}$ line were determined with respect to the O II line, and that of the Mg IX $70.6 \mathrm{~nm}$ line relative to the O III line. Although LOS bulk velocities of $\pm 15 \mathrm{~km} \mathrm{~s}^{-1}$ were found, no plume-related pattern could be identified.

\section{Discussion and conclusions}

The LOS viewing geometry for all bulk motions with small angles out of the plane of the sky allows only rather high speeds to be detected. The three-dimensional orientations of the plumes or group of plumes seen in Figs. 3 and 4 are not known in detail, but from the $17.1 \mathrm{~nm}$ images in Fig. 2 it appears as if some

\footnotetext{
17 The smallest $v_{1 / \mathrm{e}}$ of the $\mathrm{Si}^{7+}$ ions is about $30 \mathrm{~km} \mathrm{~s}^{-1}$. The highest are $39 \mathrm{~km} \mathrm{~s}^{-1}$ at $h_{01}$ and $48 \mathrm{~km} \mathrm{~s}^{-1}$ at $h_{56}$, leading to effective ion temperatures of 2.6 MK and 3.9 MK, respectively.

18 Increased $z^{\mathrm{P}} / z$ values would have no influence here, because they would be compensated by the smaller emission coefficients in plumes.
}

plume sources are in the foreground and others in the background. At an angle of $10^{\circ}$ out of the image plane, for instance, a source in the foreground would appear on the solar disk $0.015 R_{\odot}$ inside the limb, and the LOS component of a radial velocity of $\approx 50 \mathrm{~km} \mathrm{~s}^{-1}$ would become detectable with SUMER. Since no plume-related bulk motions are seen, and considering the limited depth of plumes, it is unlikely that they are accelerated to very high speeds below $140 \mathrm{Mm}$. The small integrated plume area relative to the total coronal-hole size of less than $8 \%$ is another indication that plume plasma is not a major contributor to the fast solar-wind streams. Similar conclusions have been drawn from observations of the O VI $103.2 \mathrm{~nm}$ (Wilhelm et al. 1998) and the Ne VIII $77.0 \mathrm{~nm}$ lines (Hassler et al. 1999; Wilhelm et al. 2000). For inter-plume regions, on the other hand, fore- and background contributions along the LOS can be expected to cancel each other out as far as the net Doppler shift is concerned. They cause a certain increase in the line width, which has been found not to be very significant.

The analysis of the two-dimensional maps of the four emission lines Si VIII $144.6 \mathrm{~nm}, 144.0 \mathrm{~nm}$ and MgIX $70.6 \mathrm{~nm}$, $75.0 \mathrm{~nm}$ and their radiance ratios in a coronal hole allowed a separation of the measurements into plume and inter-plume plasma parameters. The electron number densities derived here indicate rather low values in inter-plume regions. They must be regarded as more reliable than earlier ones obtained from Si VIII ratio measurements (Wilhelm et al. 1998) that used a north-south directed slit fixed at one position and might, therefore, have missed "pure" plume and lane conditions at low altitudes. The electron density is an important parameter of the coronal plasma (cf. Esser et al. 1999) and is critical for any determination of the solar-wind outflow velocities with the help of Doppler-dimming techniques (cf. Antonucci et al. 2000; Gabriel et al. 2003), as the outflow velocities deduced from such observations decrease with increasing density.

The electron density in plumes was four to five times higher than that in the environment in the range from $31 \mathrm{Mm}$ to $159 \mathrm{Mm}$ above the limb. The scale heights are larger in lanes than in plumes. The electron temperatures in inter-plume regions increase both in Fig. 8b and Table 3. The effect, although not dramatic, is in qualitative agreement with earlier line-ratio studies using many ions (Doschek et al. 1998, 2001). It is also in line with coronal freeze-in temperatures obtained from ionic chargestate measurements in the solar wind (Ko et al. 1997). The electron density and temperature distributions are strongly anticorrelated in our scan area.

Judging from the $17.1 \mathrm{~nm}$ images of EIT taken between 10 May and 6 June 2005, our plumes are above the average brightness, but are less well-defined than some other examples. It is difficult to answer the question whether or not an inversion of the plume electron temperature with height was found (cf. Table 3). Previous studies based on observations by SUMER and the Coronal Diagnostic Spectrometer (CDS, Harrison et al. 1995) detected even stronger decreases at somewhat greater altitudes (David et al. 1998; Wilhelm et al. 1998), but here it is safer to conclude that the observed low electron temperature in plumes was isothermal at the heights considered, taking into account that the decisive measurement at $h_{56}$ carries the largest uncertainty. To settle this point, longer total integration times at this and larger heights would be required in the future. Desirable would also be a full scan across the east-west extent of a coronal hole requiring an observing time of at least two days. To address the variations in elemental abundance, the strong Ne VIII line at $77.0 \mathrm{~nm}$ emitted by a high-FIP element should be included 
in the observational sequence for a comparison with the low-FIP element magnesium ${ }^{19}$.

The reasons for the height dependence of the emission coefficient and its variation in plume and inter-plume plasmas could not be identified. In particular, the systematic changes with height calls for an explanation in future studies.

The observed line widths of MgIX (and Si VIII) corroborate measurements made in 1996 (Wilhelm et al. 1998; Doschek et al. 2001), but the analysis method applied here to the twodimensional data sets renders a clearer separation into plume and lane regimes. The results that the line widths are narrower in plumes than in lanes confirm those of Antonucci et al. (1997), Hassler et al. (1997), Raju et al. (2000), and Banerjee et al. (2000a), but the difference found is larger than in most earlier studies.

Smaller velocities and effective temperatures of the silicon ions compared to the magnesium ions support earlier observations and conclusions that the various ions are independently accelerated by ion-cyclotron waves according to their charge-to-mass ratios (cf. Tu et al. 1998, and reference therein). Considering that $(Z / A)_{\mathrm{Si}^{7+}}=0.25$ and $(Z / A)_{\mathrm{Mg}^{8+}}=0.33$, our $\mathrm{Si}^{7+}$ and $\mathrm{Mg}^{8+}$ results agree with these ideas.

The effective ion temperatures increase with altitude in the inter-plume regions, but appear to be independent of height in plumes at a much lower level. Together with the low electron temperature, the conclusion can be reached that plumes are not the main regions of coronal heating.

Acknowledgements. The SUMER instrument and its operation are financed by the Deutsches Zentrum für Luft- und Raumfahrt (DLR), the Centre National d'Etudes Spatiales (CNES), the National Aeronautics and Space Administration (NASA), the European Space Agency's (ESA) PRODEX programme (Swiss contribution), and the Max-Planck-Gesellschaft (MPG). The instrument is part of SOHO, the Solar and Heliospheric Observatory of ESA and NASA. I thank the MEDOC team for their hospitality during my stay at Orsay in May 2005. Many comments on the manuscript by Werner Curdt, Eckart Marsch, Udo Schühle, Luca Teriaca, and Wing-Huen Ip are greatly acknowledged. The constructive criticism of the referee, in particular the suggestion of a forward calculation of the Si VIII radiances based on density models, improved the clarity of the paper.

\section{References}

Antonucci, E., Noci, G., Kohl, J. L., et al. 1997, ASP Conf. Ser., 118, 273 Antonucci, E., Dodero, M. A., \& Giordano, S. 2000, Sol. Phys., 197, 115 Banaszkiewicz, M., Axford, W. I., \& McKenzie, J. F. 1998, A\&A, 337, 940 Banerjee, D., Teriaca, L., Doyle, J. G., \& Wilhelm, K. 1998, A\&A, 339, 208 Banerjee, D., Teriaca, L., Doyle, J. G., \& Lemaire, P. 2000a, Sol. Phys., 194, 43 Banerjee, D., O'Shea, E., Doyle, J. G., \& Lemaire, P. 2000b, Sol. Phys., 196, 63 Bronstein, I. N., \& Semendjajew, K. A. 1985, Taschenbuch der Mathematik, 22nd Ed., Thun, Frankfurt/Main

Casalbuoni, S., Del Zanna, L., Habbal, S. R., \& Velli, M. 1999, J. Geophys. Res., 104,9947

Curdt, W., Brekke, P., Feldman, U., et al. 2001, A\&A, 375, 591

Curdt, W., Landi, E., \& Feldman, U. 2004, A\&A, 427, 1045

David, C., Gabriel, A. H., Bely-Dubau, F., et al. 1998, A\&A, 336, L90

DeForest, C. E., Hoeksema, J. P., \& Gurman, J. B., et al. 1997, Sol. Phys., 175, 393

DeForest, C. E., Plunkett, S. P., \& Andrews, M. D. 2001a, ApJ, 546, 569
DeForest, C. E., Lamy, P. L., \& Llebaria, A. 2001b, ApJ, 560, 490 Delaboudinière, J.-P., Artzner, G. E., Brunaud, J., et al. 1995, Sol. Phys., 162, 291

Del Zanna, G., \& Bromage, B. J. I. 1999, J. Geophys. Res., 104, 9753

Del Zanna, G., Bromage, B. J. I., \& Mason, H. E. 2003, A\&A, 398, 743 Domingo, V., Fleck, B., \& Poland, A. I. 1995, Sol. Phys., 162, 1 Doschek, G. A., Warren, H. P., \& Laming, J. M., et al. 1997, ApJ, 482, L109 Doschek, G. A., Laming, J. M., Feldman, U., et al. 1998, ApJ, 504, 573

Doschek, G. A., Feldman, U., Laming, J. M., et al. 2001, ApJ, 546, 559 Doyle, J. G., Mason, H. E., \& Vernazza, J. E. 1985, A\&A, 150, 69 Doyle, J. G., Teriaca, L., \& Banerjee, D. 1999, A\&A, 349, 956

Esser, R., Fineschi, S., Dobrzycka, D., et al. 1999, ApJ, 510, L63 Feldman, U., Doschek, G. A., Mariska, J. T., et al. 1978, ApJ, 226, 674 Feldman, U., Doschek, G. A., Schühle, U., \& Wilhelm, K. 1999, ApJ, 518, 500 Fludra, A., Del Zanna, G., Alexander, D., \& Bromage, B. J. I. 1999, J. Geophys. Res., 104, 9709

Gabriel, A. H., Bely-Dubau, F., \& David, C. 2002, ESA SP-477, 343 Gabriel, A. H., Bely-Dubau, F., \& Lemaire, P. 2003, ApJ, 589, 623

Gabriel, A. H., Abbo, L., Bely-Dubau, F., et al. 2005, ApJ, 635, L185 Giordano, S., Antonucci, E., Noci, G., et al. 2000, ApJ, 531, L79 Harrison, R. A., Sawyer, E. C., Carter, M. K., et al. 1995, Sol. Phys., 162, 233 Hassler, D. M., Wilhelm, K., Lemaire, P., \& Schühle, U. 1997, Sol. Phys., 175, 375

Hassler, D. M., Dammasch, I. E., Lemaire, P., et al. 1999, Science, 283, 810 Keenan, F. P., Kingston, A. E., Dufton, P. L., et al. 1984a, Sol. Phys., 94, 91 Keenan, F. P., Berrington, K. A., Burke, P. G., et al. 1984b, MNRAS, 207, 459 Ko, Y.-K., Fisk, L. A., Geiss, J., et al. 1997, Sol. Phys., 171, 345 Koutchmy, S. 1994, Adv. Space Res., 14, (4)29

Krieger, A. S., Timothy, A. F., \& Roelof, E. C. 1973, Sol. Phys., 29, 505 Laming, J. M., Feldman, U., Schühle, U., et al. 1997, ApJ, 485, 911 Lamy, P., Llebaria, A., Koutchmy, S., et al. 1997, ESA SP-404, 487 Lemaire, P., Wilhelm, K., Curdt, W., et al. 1997, Sol. Phys., 170, 105 Lites, B. W., Card, G., Elmore, D. F., et al. 1999, Sol. Phys., 190, 185 Llebaria, A., Saez, F., \& Lamy, P. 2002, ESA SP-508, 391

Mazzotta, P., Mazzitelli, G., Colafrancesco, S., \& Vittorio, N. 1998, A\&AS, 133, 403

Noci, G. 1973, Sol. Phys., 28, 403

Noci, G., Kohl, J. L., Antonucci, E., et al. 1997, Adv. Space Res., 20, 2219

Ofman, L., Nakariakov, V. M., \& Sehgal, N. 2000, ApJ, 533, 1071

Patsourakos, S., \& Vial, J.-C. 2000, A\&A, 359, L1

Pottasch, S. R. 1963, ApJ, 137, 945

Raju, K. P., Sakurai, T., Ichimoto, K., \& Singh, J. 2000, ApJ, 543, 1044 Summers, H. P., Brooks, D. H., Hammond, T. J., \& Lanzafame, A. C. 1996, Atomic Data and Analysis Structure, University of Strathclyde

Teriaca, L., Poletto, G., Romoli, M., \& Biesecker, D. A. 2003, ApJ, 588, 566 Tu, C.-Y., Marsch, E., Wilhelm, K., \& Curdt, W. 1998, ApJ, 503, 475 van de Hulst, H. C. 1950a, Bull. Astron. Inst. Netherlands, 11, 135 van de Hulst, H. C. 1950b, Bull. Astron. Inst. Netherlands, 11, 150 Wang, Y.-M. 1998, ApJ, 501, L145

Wang, Y.-M., Sheeley, N. R., Jr., Dere, K. P., et al. 1997, ApJ, 484, L75

Widing, K. G., \& Feldman, U. 1992, ApJ, 392, 715

Wilhelm, K. 1999a, ApSS, 254, 43

Wilhelm, K. 1999b, ESA SP-446, 707

Wilhelm, K. 1999c, Solar Wind Nine, AIP CP471, 269

Wilhelm, K. 2002, ISSI Scientific Report, SR-002 (ESA Publication Division), 37

Wilhelm, K., \& Bodmer, R. 1998, Space Sci. Rev., 85, 371

Wilhelm, K., Curdt, W., Marsch, E., et al. 1995, Sol. Phys., 162, 189

Wilhelm, K., Lemaire, P., Curdt, W., et al. 1997, Sol. Phys., 170, 75

Wilhelm, K., Marsch, E., Dwivedi, B. N., et al. 1998, ApJ, 500, 1023

Wilhelm, K., Dammasch, I. E., Marsch, E., et al. 2000, A\&A, 353, 749

Wilhelm, K., Inhester, B., \& Newmark, J. S. 2002, A\&A, 382, 328

Wilhelm, K., Dwivedi, B. N., Marsch, E., \& Feldman, U. 2004, Space Sci. Rev., 111,415

Young, P. R., Klimchuk, J. A., \& Mason, H. E. 1999, A\&A, 350, 286

${ }_{19}$ Observations of this type have been obtained in support of the 29 March 2006 eclipse campaigns and are being analysed at this stage. 